\title{
Recruitment of sphingosine kinase to presynaptic terminals by a conserved muscarinic signaling pathway promotes neurotransmitter release
}

\author{
Jason P. Chan, ${ }^{1}$ Zhitao Hu, ${ }^{2}$ and Derek Sieburth ${ }^{1,3}$ \\ ${ }^{1}$ Zilkha Neurogenetic Institute, University of Southern California, Los Angeles, California 90033, USA; ${ }^{2}$ Department \\ of Molecular Biology, Massachusetts General Hospital, Boston, Massachusetts 02114, USA
}

\begin{abstract}
Sphingolipids are potent lipid second messengers that regulate cell differentiation, migration, survival, and secretion, and alterations in sphingolipid signaling have been implicated in a variety of diseases. However, how sphingolipid levels are regulated, particularly in the nervous system, remains poorly understood. Here, we show that the generation of sphingosine-1-phosphate by sphingosine kinase (SphK) promotes neurotransmitter release. Electrophysiological, imaging, and behavioral analyses of Caenorhabditis elegans mutants lacking sphingosine kinase sphk-1 indicate that neuronal development is normal, but there is a significant defect in neurotransmitter release from neuromuscular junctions. SPHK-1 localizes to discrete, nonvesicular regions within presynaptic terminals, and this localization is critical for synaptic function. Muscarinic agonists cause a rapid increase in presynaptic SPHK-1 abundance, whereas reduction of endogenous acetylcholine production results in a rapid decrease in presynaptic SPHK-1 abundance. Muscarinic regulation of presynaptic SPHK-1 abundance is mediated by a conserved presynaptic signaling pathway composed of the muscarinic acetylcholine receptor GAR-3, the heterotrimeric G protein G $\alpha q$, and its effector, Trio RhoGEF. SPHK-1 activity is required for the effects of muscarinic signaling on synaptic transmission. This study shows that SPHK-1 promotes neurotransmitter release in vivo and identifies a novel muscarinic pathway that regulates SphK abundance at presynaptic terminals.
\end{abstract}

[Keywords: sphingosine kinase; mAChR; heterotrimeric G protein; Trio RhoGEF; synapse; C. elegans]

Supplemental material is available for this article.

Received January 23, 2012; revised version accepted April 11, 2012.

Acetylcholine (ACh) is a neuromodulator that is critical for cognitive, motor, and sensory processes in the brain. Many of its effects are mediated by muscarinic ACh receptors (mAChRs), the activation of which can lead to the regulation of neurotransmitter release from presynaptic terminals (Brown 2010; Drever et al. 2011). How muscarinic signaling pathways couple to presynaptic components to modulate neurotransmitter release is not well understood.

Sphingosine kinases (SphKs) are conserved lipid kinases that catalyze the conversion of sphingosine (SPH) to sphingosine-1-phosphate $(\mathrm{S} 1 \mathrm{P})$, which are potent bioactive lipids with important functions in survival, differentiation, migration, and trafficking in diverse cell types (Spiegel et al. 1998; Sarkar et al. 2005; Hannun and Obeid 2008; Yonamine et al. 2011). SphKs have also been implicated in regulating aspects of neuronal function

${ }^{3}$ Corresponding author.

E-mail sieburth@usc.edu.

Article is online at http://www.genesdev.org/cgi/doi/10.1101/gad.188003.112. and neurotransmitter release (Okada et al. 2009). Gene knockout of the SphK1 isoform impairs hippocampal long-term potentiation, spatial learning, and memory, and nociceptive processing (Mizugishi et al. 2005; Kanno et al. 2010), and selective inhibition of SphKs reduces glutamate secretion in cultured neurons (Kajimoto et al. 2007). The generation of S1P by SphKs elicits the secretion of neurotransmitters from a variety of neurons and neurosecretory cells (Alemany et al. 2001; Brailoiu et al. 2002; Pan et al. 2006; Kajimoto et al. 2007; Olivera 2008) and modulates sensory neuron excitability (Nicol 2008). In addition, SPH has been shown to have functions in synaptic vesicle (SV) exocytosis and SV cycling (Rohrbough et al. 2004; Nicol 2008; Camoletto et al. 2009; Darios et al. 2009). Furthermore, SphK1 is concentrated at presynaptic terminals in neuronal cultures (Kajimoto et al. 2007).

Cellular localization studies have revealed that SphK is transiently and rapidly recruited from cytosolic pools to cellular membranes, where S1P is produced, by a variety of extracellular and intracellular factors /Olivera and 
Spiegel 1993; Stahelin et al. 2005; Alemany et al. 2007; Bryan et al. 2008; Pitson 2011). ACh is a key extracellular factor that regulates SphK1 membrane translocation. In lung, kidney, and neuroblastoma cells, mAChR activation by muscarinic agonists can recruit SphK1 to membranes, promote S1P production, and induce SphK-dependent calcium mobilization (Meyer zu Heringdorf et al. 1998; Young et al. 2003; Pfaff et al. 2005; ter Braak et al. 2009). At synapses, SphK1 abundance is regulated by neuronal activity (Kajimoto et al. 2007). However, the molecular mechanisms underlying its synaptic regulation are not known.

Here we show that the sole Caenorhabditis elegans ortholog of mammalian $S p h K$, sphk-1, is required for proper neurotransmitter release at neuromuscular junctions (NMJs). We found that SPHK-1 is concentrated at discrete subsynaptic regions within presynaptic terminals and that presynaptic levels of SPHK-1 are critical in promoting synaptic transmission. We found that a conserved muscarinic signaling pathway composed of the $\mathrm{mAChR}$ GAR-3, the heterotrimeric G protein EGL-30, and UNC$73 /$ Trio acts in motor neurons to regulate the synaptic abundance of SPHK-1. Our results identify the regulation of SphK abundance at presynaptic terminals by muscarinic signaling as a novel mechanism by which neurotransmitter release is modulated.

\section{Results}

\section{SPHK-1 regulates presynaptic function}

C. elegans encodes a single SphK gene, sphk-1, which shares $49 \%$ and $52 \%$ amino acid similarity with mammalian SphK1 and SphK2, respectively. We previously found that RNAi knockdown of sphk-1 causes resistance to paralysis upon aldicarb treatment (Sieburth et al. 2005). Aldicarb is an acetylcholinesterase inhibitor that causes an accumulation of ACh in synaptic clefts at NMJs. Conditions or mutations that reduce ACh release confer resistance to aldicarb-induced paralysis. We examined whether sphk-1(ok1097) mutants, which lack the sphk-1 start codon and $\sim 90 \%$ of the protein-coding region (http: //www.wormbase.org), have defects in NMJ function. Mutants exhibited decreased locomotion rates and resistance to the paralytic effects of aldicarb compared with wild-type animals (Fig. 1A,B). Expression of a full-length sphk-1 cDNA transgene under the endogenous sphk-1 promoter restored both locomotion rates and aldicarb sensitivity of sphk-1 mutants to wild-type levels (Fig. $1 \mathrm{~A}, \mathrm{~B})$, indicating that the NMJ defects observed in sphk-1 mutants are due to loss of sphk-1 activity.

To determine whether sphk-1 function is required preor postsynaptically, we first examined the expression pattern of a sphk-1 transcriptional reporter in which $g f p$ expression is under the control of a rescuing sphk-1 promoter fragment. We detected GFP fluorescence in many neurons, including a majority of cholinergic and GABAergic motor neurons (Fig. 1D). In addition, GFP fluorescence was detected in body wall muscles, intestine, and hypodermis. Next, we found that transgenes expressing full-length sphk-1 cDNA specifically in the nervous system (using the snb-1 promoter) restored normal aldicarb sensitivity to sphk-1 mutants (Fig. 1E). Muscle expression of sphk-1 (using the myo-3 promoter) also rescued the aldicarb defects, although not as well as neuronal expression (Fig. 1E; Supplemental Table S1). Together, these results suggest that sphk-1 primarily acts in presynaptic neurons for aldicarb responsiveness, but sphk-1 activity in muscle also contributes to the aldicarb response. We found that sphk-1 mutants were slightly more sensitive to the paralytic effects of the muscle agonist levamisole than wild-type controls and that this sensitivity was rescued by expressing sphk-1 cDNA specifically in neurons (Fig. 1C). This suggests that there is also a modest increase in muscle excitability in sphk-1 mutants, possibly reflecting a compensatory mechanism triggered by a reduction in presynaptic activity.

We found no differences in the patterning or number of motor neurons (labeled with the acr-2 promoter-gfp reporter) in the ventral nerve cord of sphk-1 mutants compared with wild-type controls (GFP-labeled cells: $34.2 \pm 0.3$ in wild type vs. $34.8 \pm 0.4$ in sphk-1 mutants; $n=30 ; P>0.05)$. In addition, acute treatment with a selective SphK inhibitor (SKI) caused a decrease in sensitivity to aldicarb, albeit less dramatically than sphk-1 mutants (Fig. 1F). The subtle effect of SKI is presumably due to its inefficient diffusion into animals, since C. elegans skin is an efficient barrier to many compounds (Burns et al. 2010; Kage-Nakadai et al. 2010). Together, these results suggest that sphk-1 activity does not impact neuronal development or patterning, but rather regulates synaptic function.

\section{SPHK-1 promotes ACh release}

To examine ACh release directly, we recorded endogenous and electrically evoked excitatory postsynaptic currents (EPSCs) from body wall muscles. We found that the frequency and amplitude of miniature (endogenous) EPSCs (mEPSC) were similar in sphk-1 mutants and wildtype controls (Fig. 2A), indicating that spontaneous SV release and muscle responsiveness to ACh were normal. However, sphk-1 mutants displayed a $34 \%$ decrease in charge transfer following an evoked stimulus compared with wild type $(P<0.01$, Student's $t$-test) (Fig. 2B), suggesting that the total amount of neurotransmitter released in response to electrical stimulus was lower. This defect was fully rescued by re-expressing full-length sphk-1 cDNA in sphk-1 mutants. These results suggest that the defects in evoked charge transfer are likely to be due to defects in ACh release from presynaptic terminals, rather than to postsynaptic defects.

To determine whether there is an underlying defect in synapse number, synapse composition, or SV cycling in sphk-1 mutants, we analyzed changes of fluorescently tagged synaptic proteins that label a variety of different subsynaptic compartments. Motor axons form en passant synapses that can be visualized as a series of fluorescent puncta distributed along the length of axons when labeled with fluorescently tagged synaptic markers. Quantification of synaptic marker abundance at puncta (punctal 
Chan et al.

A

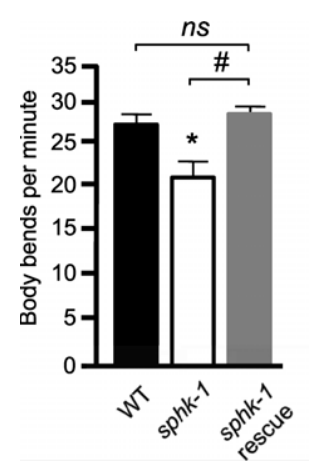

D

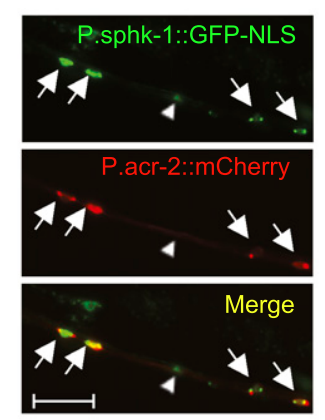

B

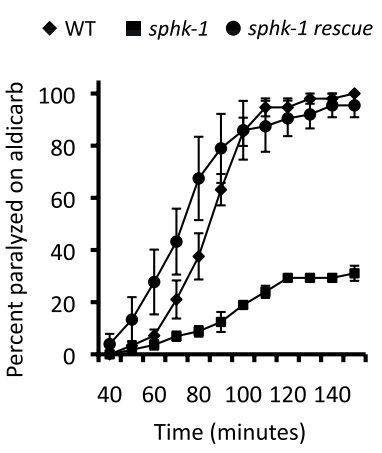

E

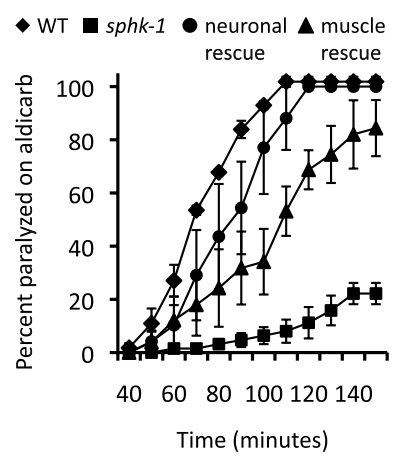

C

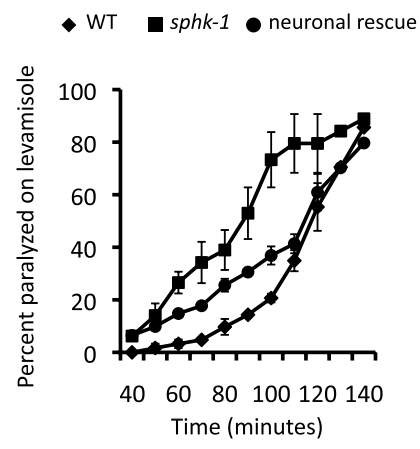

F

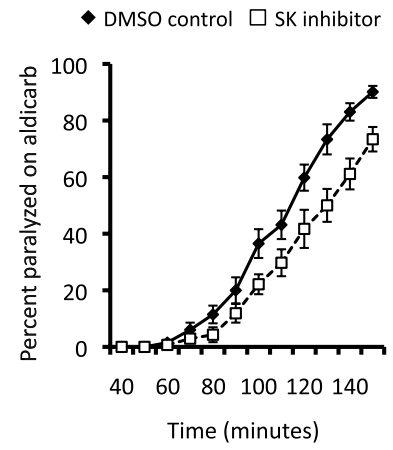

Figure 1. NMJ defects of $s p h k-1$ mutants. $(A)$ Locomotion, measured by body bends per minute, of young adult wild type (26.6 \pm 1.0$)$, sphk-1(ok1097) mutants (20.7 \pm 1.6$)$, and sphk-1 mutants expressing sphk-1 cDNA under the sphk-1 promoter (sphk-1 rescue, $28.7 \pm$ $0.8)$. Asterisks indicate values that differ significantly from wild type, and pound signs indicate differences between groups, as indicated (significance determined by ANOVA and Tukey post-hoc tests; [ns] not significant; $n=30)$. $(B)$ Rates of worm paralysis of the indicated strains upon exposure to the ACh esterase inhibitor aldicarb $(1.5 \mathrm{mM}) .(C)$ Rates of worm paralysis of the indicated strains upon exposure to the cholinergic agonist levamisole $(200 \mu \mathrm{M})$. (D) Coexpression of a sphk-1 promoter fragment driving nuclear-localized GFP (P.sphk-1-GFP-NLS) and a cholinergic neuron reporter (P.acr-2-mCherry) in ventral cord motor neurons of young adults. Arrows indicate expression in cholinergic neurons, and the arrowhead indicates expression in GABAergic neurons. Bar, $20 \mu \mathrm{m}$. (E) Rates of paralysis on aldicarb $(1.5 \mathrm{mM})$ of the indicated strains. Neuronal and muscle rescues denote transgenic sphk-1 mutants expressing fulllength sphk-1 cDNA under a pan-neuronal promoter (P.snb-1) or body wall muscle promoter (P.myo-3), respectively. $(F)$ Rates of worm paralysis upon exposure to aldicarb $(0.5 \mathrm{mM})$ after pretreatment with SKI $(50 \mu \mathrm{M})$ for $2 \mathrm{~h}$. Error bars are \pm SEM.

fluorescence), protein marker abundance in between puncta (axonal fluorescence), the size of puncta (width fluorescence), and the distance between puncta (interpunctal interval) are useful measures of the structural and functional properties of presynaptic terminals (diagram in Fig. 2C; Ch'ng et al. 2008).

We first examined whether sphk-1 mutants displayed changes in synapse number and composition by analyzing the abundance and distribution of active zone proteins (UNC-10/RIM1 and SYD-2/liprin) and periactive zone proteins (SNN-1/synapsin, APT-4/adaptin, and ITSN-1/ intersectin) in the DA class of motor neurons (using the unc-129 promoter). No changes were observed in the abundance, size, or number of UNC-10-GFP, SYD-2-GFP, SNN-1-GFP, APT-4-GFP, or ITSN-1-GFP puncta (Fig. 2D; Supplemental Table S2), suggesting that the number of synapses and the synaptic architecture of motor neurons are normal in sphk-1 mutants.

We next analyzed SV cycling at presynaptic terminals in sphk-1 mutants by examining the SV proteins SNB-1/ synaptobrevin and RAB-3/Rab3 GTPase. The intensity of
GFP-SNB-1 punctal fluorescence is correlated with the number of SVs at presynaptic terminals, whereas the intensity of diffuse GFP-SNB-1 axonal fluorescence is correlated with the abundance of synaptobrevin in the plasma membrane (Sieburth et al. 2005; Dittman and Kaplan 2006; Fernandez-Alfonso et al. 2006; Ch'ng et al. 2008). We observed significant increases in the punctal fluorescence of both GFP-SNB-1 and YFP-RAB-3 in sphk1 mutants compared with wild-type synapses (Fig. 2D). Similar changes in SNB-1 and RAB-3 distribution have been detected in mutants lacking the exocytosis protein UNC-13/Munc13 or UNC-18/Munc18 (Ch'ng et al. 2008).

Synaptic components are generated in the cell body and selectively targeted to axonal and dendritic specializations (Ou and Shen 2011). If the increase in GFP-SNB-1 at presynaptic terminals in sphk-1 mutants could be explained by changes in biogenesis or trafficking of SVs, then we would also expect alterations in GFP-SNB-1 fluorescence in cell bodies or dendrites in motor neurons in mutants. However, we observed no changes in GFPSNB-1 fluorescence intensity in cell bodies or dendrites in 
A
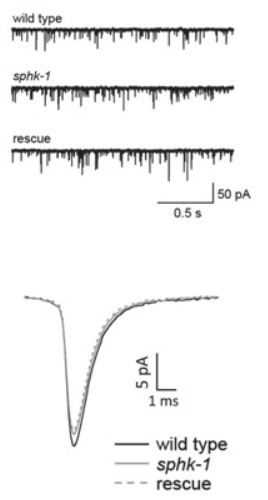

C

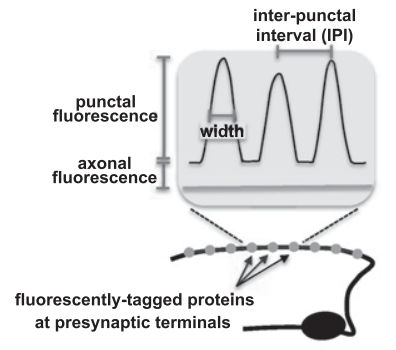

B
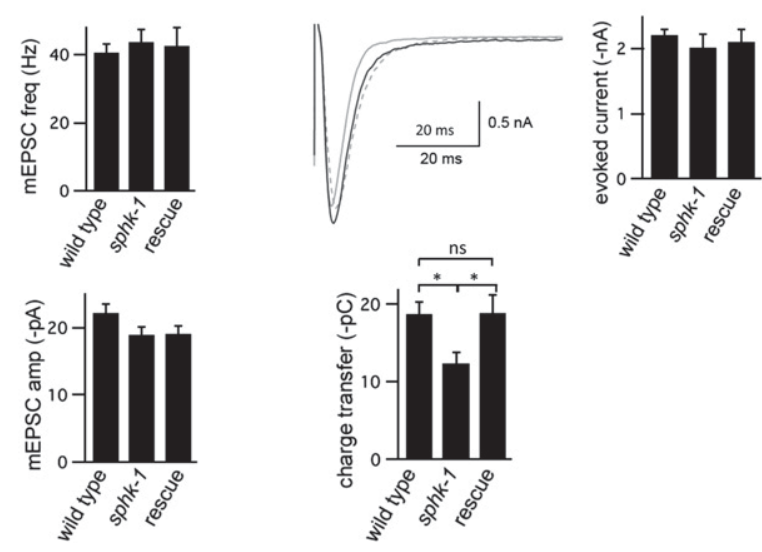

D

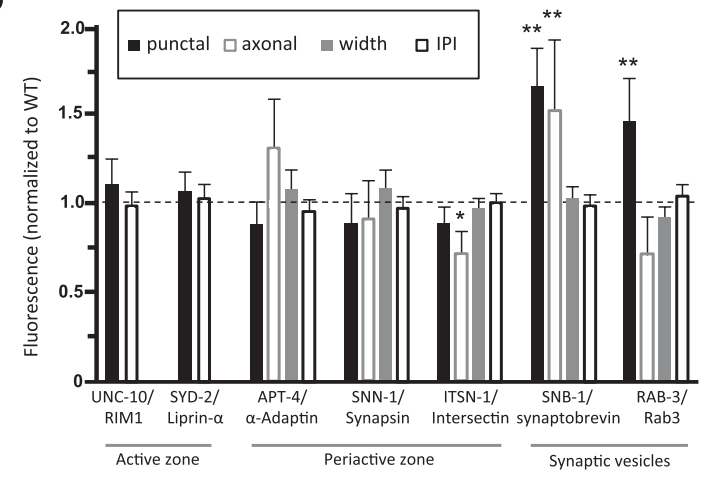

$\mathrm{E}$
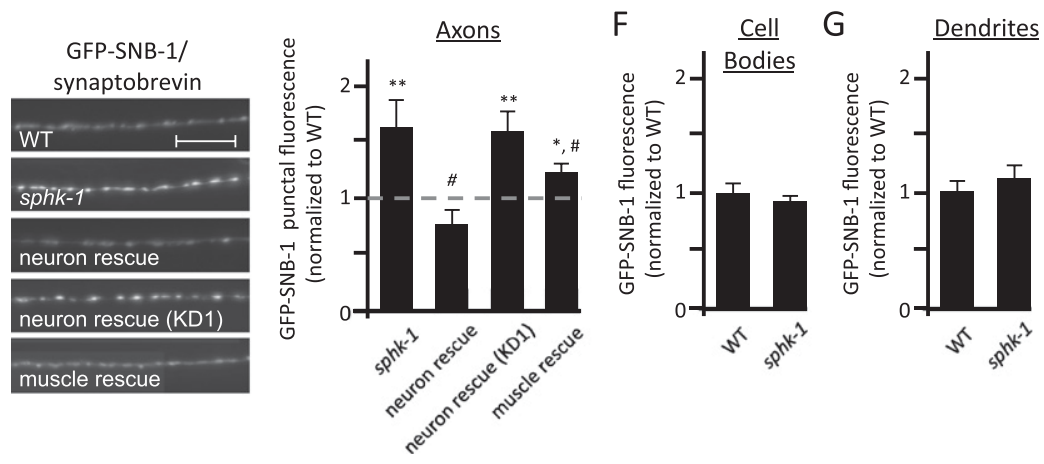

Figure 2. Synaptic transmission and vesicle cycling defects in sphk-1 mutants. (A) Quantification of endogenous mEPSCs in wild-type (WT), sphk-1 mutant, and P.sphk-1 rescue animals. (Top left) Representative traces of mEPSCs. (Top right) Quantification of the mEPSC frequency: wild-type animals $(40.7 \mathrm{~Hz} \pm 2.5 \mathrm{~Hz} ; n=5)$, sphk-1 mutant animals $(48.8 \mathrm{~Hz} \pm 3.6 \mathrm{~Hz} ; n=9)$, and P.sphk-1 rescue animals $(42.5 \mathrm{~Hz} \pm 5.6 \mathrm{~Hz} ; n=6)$. (Bottom left) Representative traces of the average mEPSC. (Bottom right) Quantification of mEPSC amplitude: wild-type animals $(22.2 \mathrm{pA} \pm 1.3 \mathrm{pA})$, sphk-1 mutant animals $(19.0 \mathrm{pA} \pm 1.2 \mathrm{pA})$, and P.sphk-1 rescue animals $(19.1 \mathrm{pA} \pm 1.2 \mathrm{pA}) .(B)$ Quantification of evoked EPSCs in wild-type, sphk-1 mutant, and P.sphk-1 rescue animals. (Top left) Representative traces of evoked current. (Top right) Quantification of the peak amplitude: wild-type animals (2.2 nA $\pm 0.1 \mathrm{nA} ; n=8)$, sphk-1 mutant animals $(2.0 \mathrm{nA} \pm$ $0.2 \mathrm{nA} ; n=8)$, and P.sphk-1 rescue animals (2.1 nA $\pm 0.2 \mathrm{nA} ; n=5)$. (Bottom left) Quantification of total charge transfer: wild-type animals $(18.8 \mathrm{pC} \pm 1.6 \mathrm{pC})$, sphk-1 mutant animals $(12.4 \mathrm{pC} \pm 1.4 \mathrm{pC})$, and P.sphk-1 rescue animals $(18.9 \mathrm{pC} \pm 2.3 \mathrm{v})$. Asterisks indicate values that differ between the groups shown; $\left(^{\star}\right) P<0.05$. $(C)$ Schematic of the quantification method used to determine the abundance of fluorescently tagged proteins at synapses (punctal) and between synapses (axonal), synapse size (width), and synapse number (interpunctal interval [IPI]) of DA motor neurons. $(D)$ Analysis of the abundance of a panel of YFP- or GFP-tagged SV proteins in sphk-1 mutants, normalized to wild type (dotted line). (E, left) Representative images of GFP-tagged synaptobrevin (GFP-SNB-1) in DA motor neurons in the indicated strains. Motor neuron rescue was performed by expressing cDNA for full-length or a kinase-dead (KD1) sphk-1 under the unc-129 promoter and muscle rescue under the myo-3 promoter. (Right) Quantification of GFP-SNB-1 punctal fluorescence, normalized to wild type (dotted line). Bar, $10 \mu \mathrm{m} .(F, G)$ Quantification of GFP-SNB-1 fluorescence in cell bodies $(F)$ and punctal fluorescence in dendrites $(G)$ of wild type and sphk-1 mutants. Values are normalized to wild type. For all quantification, asterisks indicate values that differ significantly from wild type, and pound signs indicate values that differ from sphk-1(ok1097) mutants $\left(\left[{ }^{\star}\right] P<\right.$ 0.05; [ $\left.{ }^{\star \star}\right] P<0.005$; [\#] $P<0.05$; one-way ANOVA and Tukey's post-hoc tests; [ns] not significant). 
sphk-1 mutants (Fig. 2F,G), suggesting that SPHK-1 functions to promote the release of SVs from presynaptic terminals.

The increase in synaptic fluorescence of GFP-SNB-1 observed in sphk-1 mutants was completely rescued when sphk-1 cDNA was expressed specifically in motor neurons (using the unc-129 promoter) (Fig. 2E). Interestingly, we observed that re-expression of sphk-1 cDNA in body wall muscles partially rescued the $s p h k-1$ mutant phenotype. Thus, sphk-1 functions both cell-autonomously in motor neurons and non-cell-autonomously in muscles to promote SV release.

\section{SPHK-1 functions at presynaptic terminals}

Given the role of SPHK-1 in regulating neurotransmitter secretion, we next determined whether it was concentrated at sites of SV release. We examined the subcellular localization of a fully functional fusion of GFP-tagged SPHK-1 (SPHK-1-GFP) in cholinergic motor neurons. Fluorescence was observed in cell bodies in addition to axons, where SPHK-1-GFP adopted a highly punctate pattern of fluorescence (Fig. 3). SPHK-1-GFP puncta were observed near the SV proteins SNB-1/synaptobrevin and RAB-3/Rab3 (Fig. 3A; data not shown), indicating that SPHK-1 is enriched at presynaptic terminals. Interestingly, not all SNB-1 puncta were associated with a corresponding SPHK-1 punctum, and SPHK-1 puncta were generally much smaller and more distinct than SV puncta. SPHK-1-GFP axonal fluorescence (fluorescence between puncta) was not detectable above background, suggesting that there is very little nonsynaptic SPHK-1 in these neurons. To test whether SPHK-1 directly associates with SVs, we examined the localization of SPHK-1GFP in mutants with altered SV distribution. SPHK-1GFP remained punctate in mutants lacking UNC-104/ KIF1A (Fig. 3B), a kinesin motor protein necessary for SV axonal transport (Hall and Hedgecock 1991). In addition, the intensity, size, and number of SPHK-1-GFP puncta were not altered in mutants with increased (unc-13) or decreased (unc-57/endophilin) presynaptic SV pools (Ch'ng et al. 2008), indicating that localization of SPHK-1 in axons is not dependent on SVs (Fig. 3B; Supplemental Table S3). We next tested whether SPHK-1 localizes to other subsynaptic regions within presynaptic terminals. We found that SPHK-1 puncta were mostly apposed to but did not colocalize with the active zone marker UNC10/RIM1, which marks sites of SV release (Fig. 3C), or the dense core vesicle marker NLP-21/neuropeptide-like protein (Fig. 3D). SPHK-1 puncta colocalized more consistently, although not completely, with the periactive zone proteins GSNL-1/gelsolin, ITSN-1/intersectin, and SNN1 /synapsin (Fig. 3E; data not shown). Periactive zones are regions of presynaptic membranes that contain proteins involved in endocytosis and actin regulation and may be specialized for SV recycling. Together, these data suggest that SPHK-1 is not directly associated with SVs but instead is concentrated on regions of presynaptic membranes that are likely to be close to periactive zones.

If SPHK-1-GFP localization at presynaptic terminals is important for its function in promoting ACh release, then we expect that altering the abundance of SPHK-1 at synapses would cause corresponding changes in synaptic transmission. Consistent with this idea, we found that
A

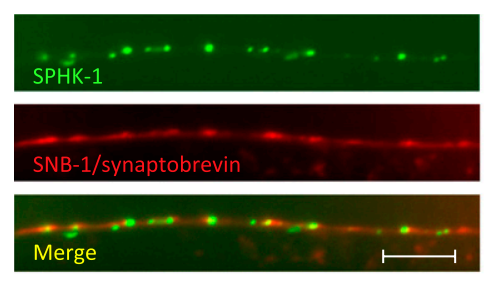

D

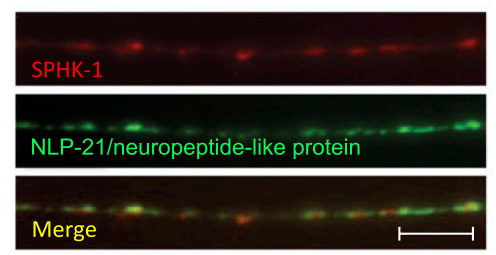

B

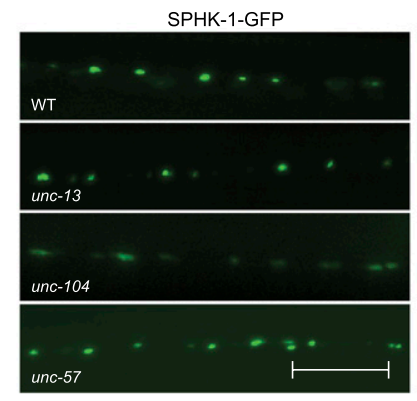

$\mathrm{E}$

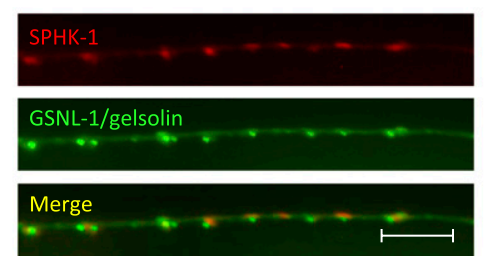

C

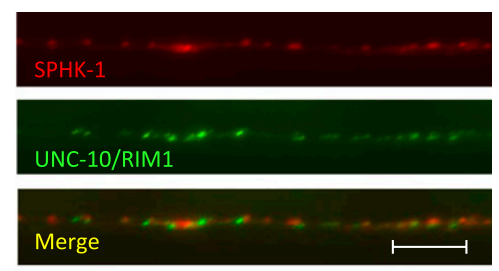

Figure 3. SPHK-1 localizes to presynaptic terminals. (A) Representative images showing the localization pattern of SPHK-1-GFP and SNB-1/synaptobrevin (mCherry-SNB-1) in adult DA axons. (B) Images of SPHK-1-GFP distribution in mutants lacking the SV trafficking protein unc-104/kinesin KIF1A or the SV cycling proteins unc-13/Munc13 and unc-57/endophilin. (C-E) Representative images showing the localization of SPHK-1-mCherry with UNC-10/RIM1 (UNC-10-GFP), NLP-21/neuropeptide (NLP-21-YFP), or GSNL-1/gelsolin (GSNL-1-GFP). Bar, $10 \mu \mathrm{m}$. 
animals overexpressing wild-type SPHK-1 transgenes displayed significantly increased sensitivity to aldicarb compared with nontransgenic controls (Fig. 4A). We confirmed that animals overexpressing SPHK-1 had increased SPHK-1 abundance at presynaptic terminals by comparing SPHK-1-GFP punctal fluorescence from animals expressing one copy of the transgene (from heterozygotes) to two copies of the transgene (from homozygotes). We found an increase in peak fluorescence of $\sim 214 \%(P<$ 0.001 , Student's $t$-test) in animals expressing two copies of the SPHK-1-GFP transgene. These results suggest that increasing SPHK-1 dosage results in corresponding increases in both SPHK-1 synaptic abundance and aldicarb hypersensitivity.

Conversely, we predict that decreasing SPHK-1 abundance at synapses would also alter synaptic transmission. To test this, we interfered with the ability of SPHK-1 to translocate to synapses by mutating its calcium/calmodulin (CaM)-binding site. Mutations in the CaM-binding site of mammalian SphK have been reported to decrease translocation of SphK to membranes but to have no effect on its catalytic activity (Sutherland et al. 2006; Jarman et al. 2010). We found that GFP-tagged SPHK-1 transgenes lacking the functional CaM-binding site [SPHK-1( $\Delta \mathrm{CaM})$ GFP] were expressed at levels in the cell bodies of neurons similar to wild-type transgenes [cell body fluorescence was $2280 \pm 243$ arbitrary units for SPHK-1(WT)-GFP and $2561 \pm 222$ arbitrary units for SPHK-1(DCaM)-GFP; $n=20]$. However, peak fluorescence at synapses was significantly decreased compared with SPHK-1-GFP controls (Fig. 4B,C). Importantly, SPHK-1( $\Delta \mathrm{CaM})$ transgenes failed to rescue the aldicarb resistance of $s p h k-1$ mutants (Fig. 4A, hatched bars), implying that the CaM-binding site is necessary for both the translocation and function of SPHK-1 at synapses. Interestingly, SPHK-1( $\Delta \mathrm{CaM})$-GFP puncta were significantly wider than SPHK-1 puncta $(0.89 \pm 0.02 \mu \mathrm{m}$ for wild type and $1.24 \pm 0.05 \mu \mathrm{m}$ for $\Delta \mathrm{CaM}_{;} P<0.001$ ) (Fig. 4B,C), and colocalization with SPHK-1-mCherry revealed that SPHK-1 $(\Delta \mathrm{CaM})-\mathrm{GFP}$ puncta extended beyond the regions normally occupied

A

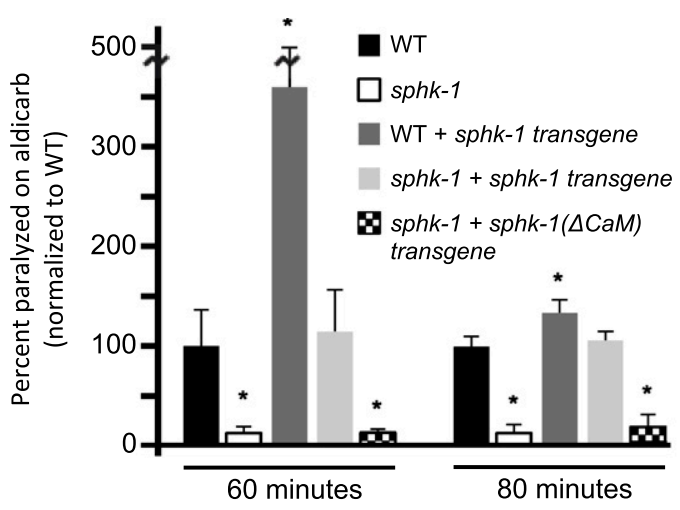

B

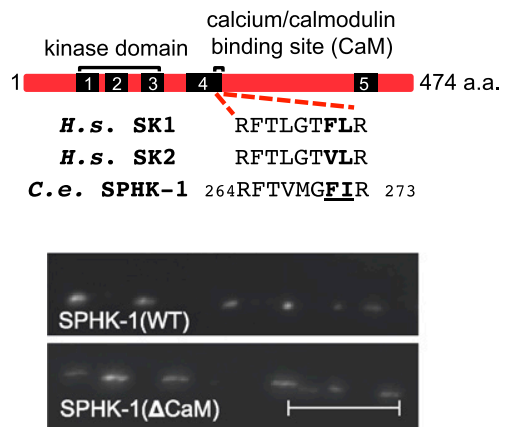

C

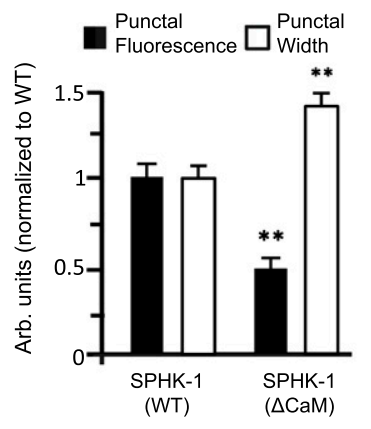

D

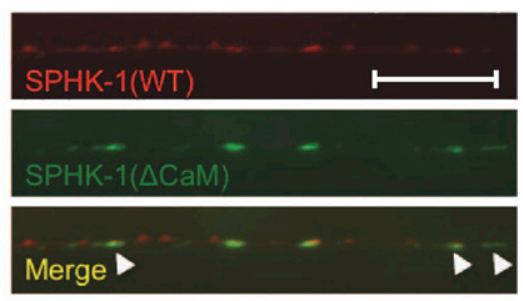

Figure 4. Levels of SPHK-1 at synapses affect aldicarb responsiveness. (A) Rates of worm paralysis at 60 and 80 min in aldicarb (1.0 $\mathrm{mM}$ ) of wild-type (WT) animals, sphk-1 mutants, transgenic wild-type animals expressing sphk-1 cDNA under the pan neuronal snb-1 promoter (wild-type + sphk-1 transgene), and sphk-1 mutants expressing either wild-type (sphk-1 + sphk-1 transgene) or mutated [sphk$1+\operatorname{sphk}-1(\Delta C a M)$ transgene] sphk-1 cDNA under the pan neuronal snb-1 promoter. $(B$, top $)$ Schematic of SPHK-1 indicating the five conserved domains (C1-C5) and the CaM-binding site. The sequence mutated in SPHK-1( $\Delta \mathrm{CaM})$ is underlined. (Bottom) Representative images of GFP tagged to wild-type SPHK-1 [SPHK-1(WT)] or to SPHK-1 with its CaM-binding site mutated [SPHK-1( $\Delta$ CaM)]. (C) Quantification of the punctal fluorescence and width in wild-type animals expressing either SPHK-1(WT)-GFP or SPHK-1( $\Delta$ CaM)-GFP transgenes. $\left(^{\star \star}\right) P<0.005$, Student's $t$-test. $(D)$ Images showing the localization pattern of SPHK-1(WT)-mCherry with SPHK-1( $\Delta$ CaM)GFP coexpressed in DA neurons. Arrowheads indicate SPHK-1(DCaM)-GFP puncta with greater widths compared with SPHK-1mCherry. Bars, $10 \mu \mathrm{m}$. Error bars are \pm SEM. 
by SPHK-1(WT)-mCherry (Fig. 4D). Together, these results indicate that maintaining a highly concentrated and spatially restricted distribution of SPHK-1 at synaptic (or other) membranes may be critical for its function.

\section{S1P generation by SPHK-1 regulates neurotransmission}

To determine whether the synaptic defects seen in sphk-1 mutants arise from altered SPH or S1P levels, we examined the effects of catalytically inactive sphk-1 on SPHK-1 localization and function. We generated two kinase-dead (KD) sphk-1 variants that contain amino acid substitutions in critical regions of the catalytic domain: SPHK-1(KD1) contains a five-amino-acid substitution in a conserved region (SGDGL to AAAAA) in the kinase domain reported to be critical for nucleotide binding and activity of mammalian SphK1 (Pitson et al. 2002), and SPHK-1(KD2) contains a GSGN-to-DDDD mutation, which has been shown to block kinase activity in Drosophila SphK (Yonamine et al. 2011). Transgenes expressing either of the kinase-dead variants failed to rescue the aldicarb resistance defects of sphk-1 mutants (Supplemental Fig. S1). In addition, we found that although the SPHK-1(KD1) variant adopted a punctate pattern of fluorescence in axons similar to wild-type SPHK-1-GFP (data not shown), it failed to rescue the SV cycling defects of sphk-1 mutants (Fig. 2E). Thus, the kinase activity of SPHK-1 is necessary to restore proper aldicarb responsiveness and SV cycling to sphk-1 mutants.

Analysis of whole-worm extracts by mass spectrometry has shown a 10-fold increase in SPH levels in sphk-1 mutants (Menuz et al. 2009). However, for reasons that are not entirely clear, S1P and its derivatives have been difficult to reliably detect from these extracts (Menuz et al. 2009; D Sieburth, unpubl.). To circumvent this limitation, we examined genetic interactions between sphk-1 and hyl-1/ceramide (CER) synthase mutants. CER synthase is an enzyme that converts SPH to CER (Fig. 5A). hyl-1 mRNA is expressed in C. elegans motor neurons (Fox et al. 2005). Like sphk-1 mutants, hyl-1/CER synthase mutants have increased SPH levels, as determined by mass spectrometry (Menuz et al. 2009). If increased SPH levels cause the synaptic transmission defects associated with sphk-1 mutants, then hyl-1 mutants should display a reduction in locomotion rates similar to sphk-1 mutants.
However, we found that hyl-1 mutants displayed significantly increased locomotion rates compared with wildtype animals (Fig. 5B). These results indicate that high SPH levels are not likely to contribute to the defects seen in sphk-1 mutants. On the other hand, if S1P production were necessary for synaptic function, then we would expect sphk-1 mutations to suppress the locomotion defects seen in hyl-1 mutants because the double mutants can no longer make S1P. Consistent with this, we found that sphk-1;hyl-1 double mutants had locomotion rates that were similar to sphk-1 mutants (Fig. 5B). Together, these results suggest that the synaptic transmission defects of sphk-1 mutants are likely to result from lack of S1P production by SPHK-1.

\section{Endogenous ACh regulates SPHK-1 abundance at synapses}

In C. elegans, two opposing heterotrimeric G proteins modulate neurotransmission at the NMJ: egl-30/G $\alpha \mathrm{q}$ and goa-1/G $\alpha \mathrm{O}$, which have excitatory and inhibitory effects, respectively (Perez-Mansilla and Nurrish 2009). However, the G-protein-coupled receptors (GPCRs) activating these $G$ proteins in neurons and the mechanisms coupling $G$ protein activation to neurotransmitter release are not well understood. We tested whether SPHK-1 might be a downstream component of either of these pathways in motor neurons. We first examined whether activation of these G proteins using GPCR agonists could alter the synaptic abundance of SPHK-1-GFP at presynaptic terminals. We found that acute $(2-h)$ treatment of animals with arecoline, a muscarinic agonist that activates egl-30/ $\mathrm{G} \alpha \mathrm{q}$ (Lackner et al. 1999), caused a significant increase in SPHK-1-GFP punctal fluorescence (Fig. 6A) but did not alter punctal width or density (Supplemental Table S3). In contrast, serotonin treatment, which activates goa-1/G $\alpha 0$ (Nurrish et al. 1999), had no effect on any parameter measured for SPHK-1-GFP localization or abundance (Fig. 6A; Supplemental Table S3).

Previous studies in $C$. elegans have suggested that arecoline treatment causes aldicarb hypersensitivity by promoting the recruitment of the UNC-13 to synapses (Lackner et al. 1999). Therefore, we wondered whether the alterations in SPHK-1 abundance following arecoline treatment could be a secondary consequence of areco-
A

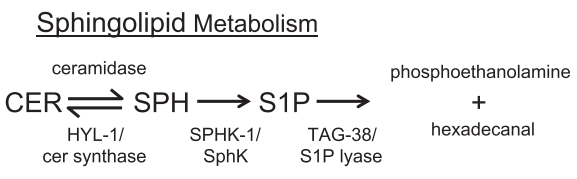

B

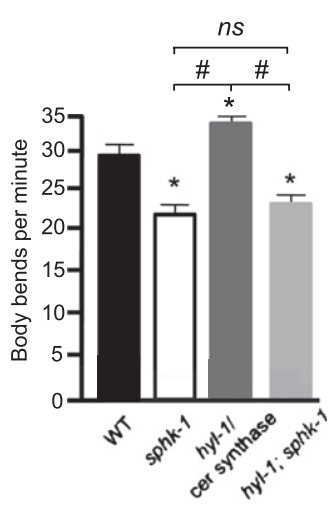

Figure 5. S1P is necessary to promote neurotransmission. (A) Diagram of the sphingolipid metabolism pathway and its enzymes. (B) Quantification of body bends per minute in indicated strains (wild type [WT], $28.5 \pm 1.2$; sphk-1, $21.1 \pm 0.8$; hyl-1, $32.3 \pm 0.8$; hyl-1;sphk-1, $22.9 \pm 0.8 ;\left[^{\star}\right] P<$ 0.01 compared with wild type; [\#] $P<0.01$ compared between the indicated groups; significance was determined by ANOVA and Tukey's post-hoc tests). Bars, $10 \mu \mathrm{m}$. Error bars are \pm SEM. 
A

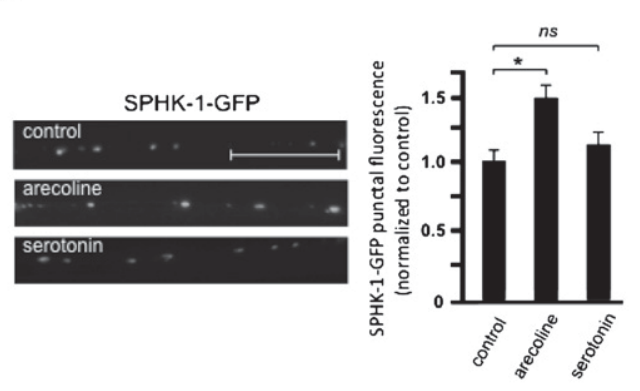

B

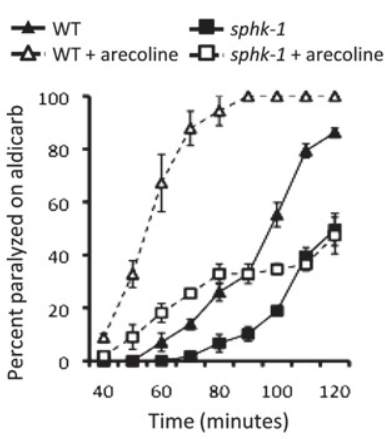

C

D
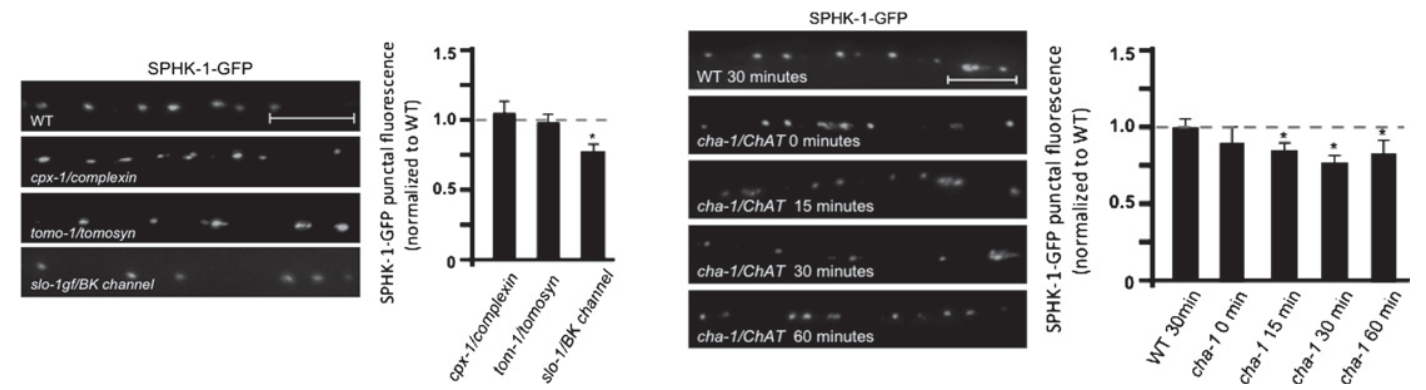

E

$\mathrm{F}$
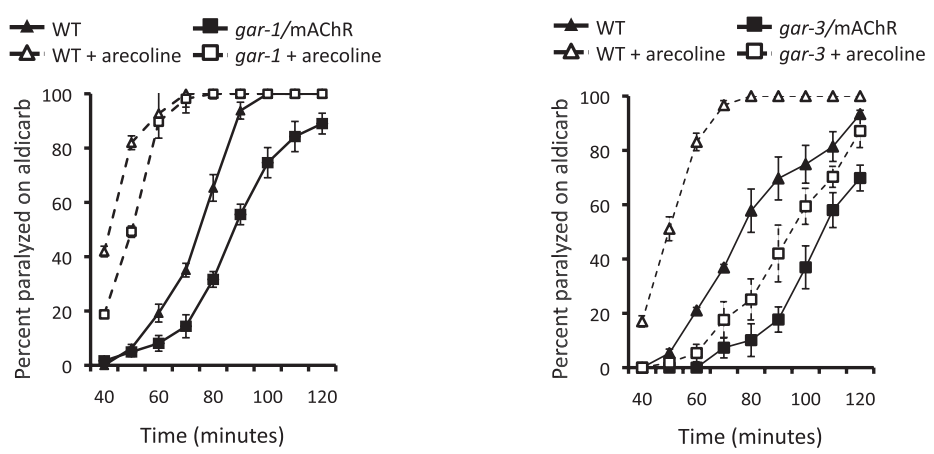

G
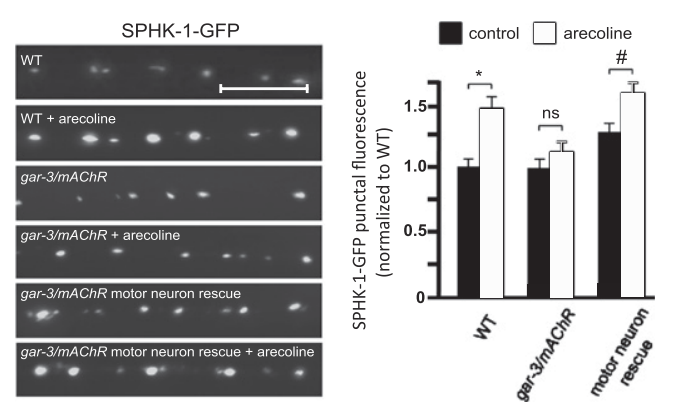

Figure 6. GAR-3 mAChR activation in motor neurons regulates SPHK-1 abundance. $(A$, left) Representative images of SPHK-1-GFP following $2 \mathrm{~h}$ of exposure to plates with M9 (control), arecoline (15 mM), or serotonin (10 mM). (Right) Quantification of the SPHK-1GFP punctal fluorescence (control vs. arecoline, $\left.{ }^{\star}\right] P<0.05$; control vs. serotonin, $P>0.05$; ANOVA and Tukey's post-hoc tests). (B) Rates of paralysis on aldicarb $(1.0 \mathrm{mM})$ for wild-type animals compared with sphk-1 with or without arecoline treatment. $(C, D)$ Representative images (left) and quantification (right) of SPHK-1-GFP in the indicated strains. Quantification of SPHK-1-GFP punctal fluorescence was normalized to wild type (dotted line; $\left.{ }^{\star}\right] P<0.05$; Student's $t$-test). $(E, F)$ Rates of paralysis on aldicarb (1.0 mM) for wild-type animals compared with gar-1/mAChR $(E)$ or gar-3/mAChR $(F)$ mutants with and without acute arecoline treatment. $(G)$ Representative images (left) and quantification (right) of changes in SPHK-1-GFP punctal fluorescence following arecoline treatment (2 h) in the indicated strains. Neuronal rescue denotes gar-3 mutants expressing a P.unc-129-gar-3 cDNA transgene. Asterisks indicate values that differ significantly from wild type $\left(\left[{ }^{\star}\right] P<0.01\right.$; [\#] $P<0.01$; ANOVA and Tukey's post-hoc tests). Bars, $10 \mu \mathrm{m}$. Error bars are \pm SEM for aldicarb curves or propagated SEM for quantitative imaging. 
line's effect on presynaptic activity. To test this idea, we examined whether SPHK-1-GFP fluorescence changes in mutants with altered ACh secretion from NMJs or altered electrical activity. We found no significant changes in SPHK-1-GFP punctal fluorescence in mutants that either increase (tomo-1/tomosyn or $c p x-1 /$ complexin) (Fig. 6C) or decrease (unc-13/Munc13 or unc-18/Munc18) (Fig. 7B; Supplemental Table S3) ACh secretion (Richmond et al. 1999; Weimer et al. 2003; Madison et al. 2005; Gracheva et al. 2006; Vashlishan et al. 2008; Hobson et al. 2011; Martin et al. 2011). In contrast, mutants expressing a constitutively active slo-1/BK potassium channel that inhibits neuronal activity (Richmond et al. 2001; Q Liu et al. 2007) displayed reduced SPHK-1-GFP punctal fluorescence (Fig. 6C). Thus, the effect of arecoline treatment on SPHK-1 abundance is not a secondary effect of alterations in SV release, but rather is likely to reflect the direct recruitment of SPHK-1 to motor synapses by muscarinic signaling. In addition, the recruitment of
SPHK-1 presynaptic terminals is likely to be dependent on presynaptic activity.

Does endogenously produced ACh regulate SPHK-1 abundance? To test this, we examined animals deficient in ACh biosynthesis for effects on SPHK-1-GFP punctal fluorescence. cha-1/ChAT encodes the only C. elegans ortholog of choline acetyltransferase. cha-1/ChAT-null mutants are born paralyzed and die due to starvation, presumably due to the lack of ACh production (Rand 1989). However, cha-1/ChAT (p1182) temperature-sensitive mutants are viable but become paralyzed within 15 min of being shifted from $20^{\circ} \mathrm{C}$ to $25^{\circ} \mathrm{C}$ (Rand 1989). We found that shifting cha-1/ChAT mutants expressing SPHK-1-GFP transgenes for 15,30 , or $60 \mathrm{~min}$ to $25^{\circ} \mathrm{C}$ resulted in significant decreases in SPHK-1-GFP punctal fluorescence compared with temperature-shifted wildtype controls (Fig. 6D). In contrast, cha-1/ChAT mutants that were not temperature-shifted were not significantly different from wild-type controls (Fig. 6D). Together,
A

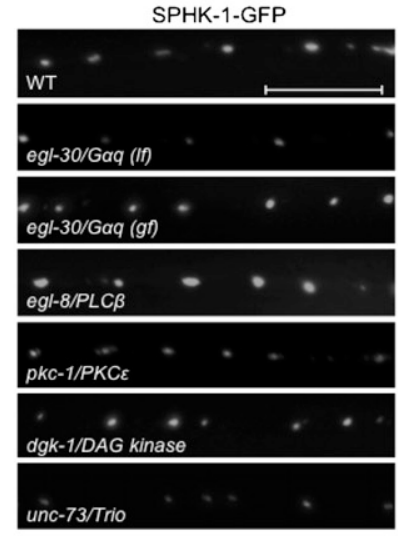

C

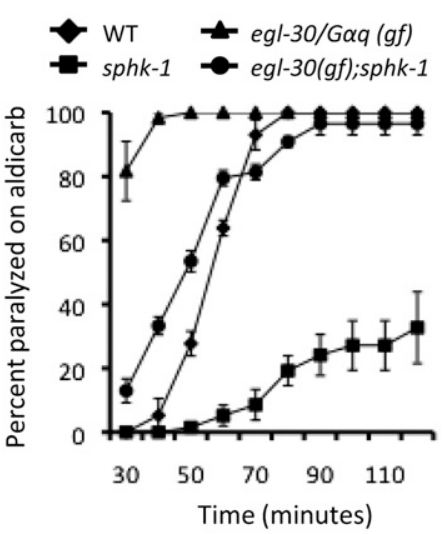

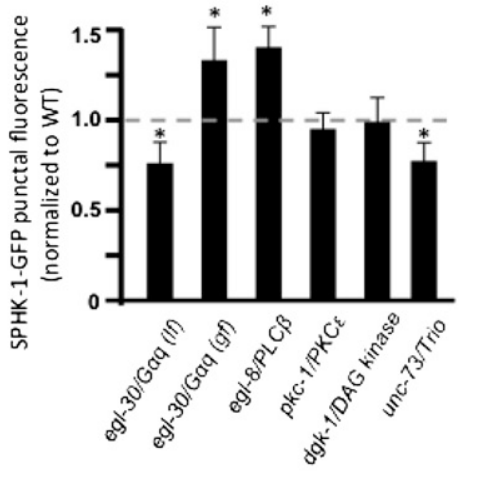

D
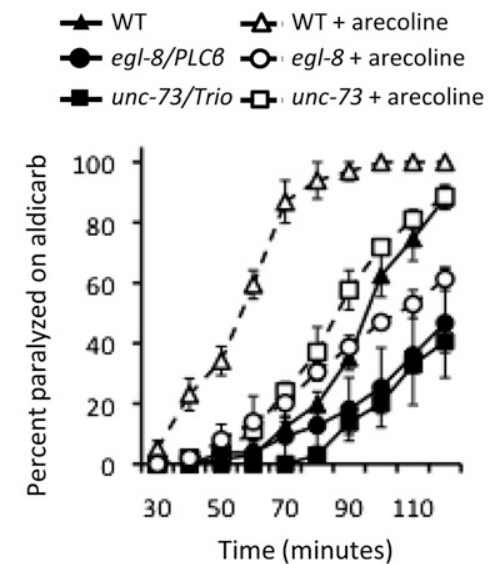
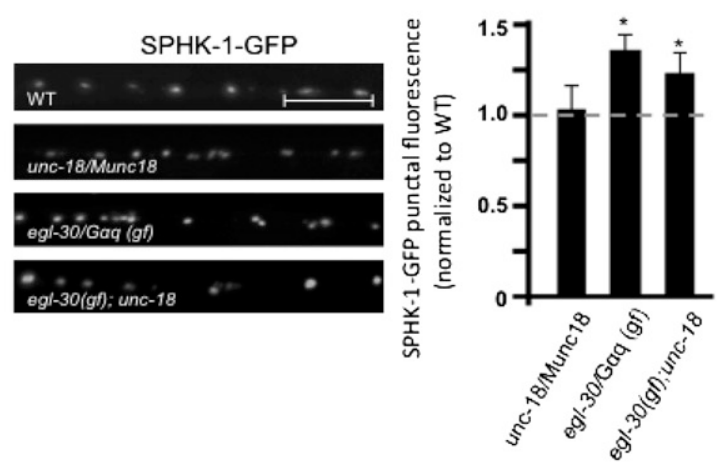

E

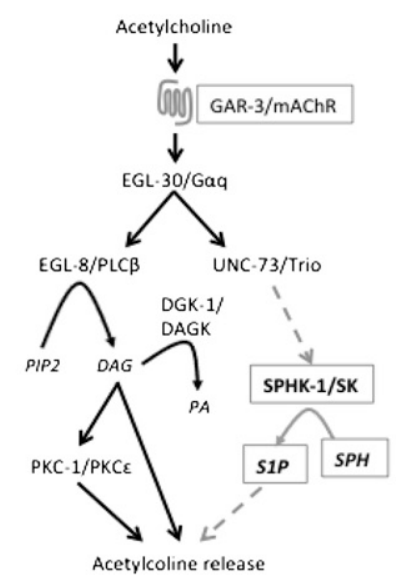

Figure 7. Regulation of SPHK-1 synaptic abundance by the G $\alpha \mathrm{q}$ signaling pathway. $(A, B)$ Representative images and quantification of SPHK-1-GFP fluorescence of DA motor axons in the indicated strains. $(C, D)$ Rates of paralysis on aldicarb $(1.0 \mathrm{mM})$ for the indicated strains. For unc-73, the ce362 mutation was used. The indicated animals were pretreated with arecoline (15 mM in M9) or control (M9) for $2 \mathrm{~h}$. (E) Model of the muscarinic signaling pathway in C. elegans cholinergic motor neurons that regulates SPHK-1 synaptic abundance and neurotransmitter release. Established model of the G-protein pathway in C. elegans motor neuron (Perez-Mansilla and Nurrish 2009), with novel components identified in this study shown in boxes. For quantification of SPHK-1-GFP, values are normalized to wild type (represented by dotted line); $\left(^{\star}\right) P<0.05$ (Student's $t$-test). Bars, $10 \mu \mathrm{m}$. Error bars are \pm SEM for aldicarb curves, or \pm propagated SEM for quantitative imaging. 
these results strongly support the idea that ACh can regulate SPHK-1 abundance bidirectionally: Muscarinic agonists can increase SPHK-1 synaptic abundance, whereas reductions in endogenously produced ACh decrease SPHK-1 synaptic abundance. Furthermore, these results show that ACh can modify SPHK-1 abundance at rapid timescales (in as little as $15 \mathrm{~min}$ ) and therefore is likely to act post-translationally to regulate synaptic SPHK-1 levels.

\section{gar-3/mAChR mediates the effects of ACh and acts cell-autonomously}

To identify the mAChR that mediates the effects of arecoline on SPHK-1 abundance, we tested the effects of mAChR family members on SPHK-1 recruitment. gar-1 and gar-3 encode mAChRs homologous to M1, M3, and M5 classes of mammalian muscarinic receptors and are predicted to couple to egl-30/G $\alpha \mathrm{q}$. We found that both gar-1 and gar-3 putative null mutants were weakly resistant to paralysis by aldicarb (Fig. 6E,F). However, gar-3/ $\mathrm{mAChR}$ mutants were mostly unresponsive to the effects of arecoline, whereas gar- $1 / \mathrm{mAChR}$ mutants responded similarly to wild-type controls. Interestingly, gar- $3 / \mathrm{mAChR}$ mutations also blocked the arecoline-induced increase in SPHK-1-GFP punctal fluorescence (Fig. 6G). These results suggest that gar-3/mAChR is required for the muscarinic agonist-mediated effects on aldicarb hypersensitivity and SPHK-1 synaptic recruitment. gar-3/mAChR mRNA is enriched in cholinergic motor neurons (Fox et al. 2005), and transcriptional reporters show gar-3/mAChR expression in muscles, the pharynx, motor neurons, and some neurons in the male tail (Steger and Avery 2004; Y Liu et al. 2007). We found that expression of gar-3/mAChR cDNA specifically in motor neurons (using the unc-129 promoter) was sufficient to fully restore arecoline-induced increases in SPHK-1-GFP punctal fluorescence to gar-3/mAChR mutants (Fig. 6G). Thus, gar-3/mAChR acts cell-autonomously in motor neurons to recruit SPHK-1 to release sites in response to cholinergic agonists.

If SPHK-1 recruitment to presynaptic terminals by muscarinic signaling were important for the effects of GAR-3 activation on neurotransmission, then we would expect that the aldicarb hypersensitivity caused by arecoline treatment would be attenuated in sphk-1 mutants. Consistent with this idea, we found that sphk-1 mutants were significantly less responsive to arecoline treatment compared with wild-type controls (Fig. 6B). Taken together, our data indicate that the recruitment of SPHK-1 by arecoline is functionally important for the effects of muscarinic signaling on synaptic transmission.

\section{Gaq-Trio signaling regulates synaptic SPHK-1} abundance

GAR-3/mAChR has been shown to activate the heterotrimeric G protein EGL-30/Goq in certain cells in C. elegans (Steger and Avery 2004; Y Liu et al. 2007); thus, we predicted that GAR-3/mAChR couples to EGL-30/G $\alpha$ q in motor neurons. Consistent with this, egl-30/Gaq lossof-function mutations significantly reduced the synaptic fluorescence intensity of SPHK-1-GFP compared with wild-type controls (Fig. 7A). Conversely, constitutively active egl-30/G $\alpha \mathrm{q}$ mutations [egl-30(gf)] significantly increased SPHK-1-GFP punctal fluorescence, indicating that egl-30/G $\alpha \mathrm{q}$ signaling regulates SPHK-1 synaptic abundance in motor neurons. The effects of egl-30(gf) on SPHK-1 abundance were not blocked by unc-18/Munc18 mutations (Fig. 7B), confirming that SPHK-1 synaptic recruitment is not a secondary effect of egl-30/Gaq signaling on SV exocytosis. We also found that sphk-1 mutations partially block the aldicarb hypersensitivity of egl-30(gf) mutants (Fig. 7C), confirming that sphk-1 is a functionally relevant downstream target of the GAR-3/ mAChR-EGL-30/G $\alpha q$ pathway in these neurons.

EGL-30/G $\alpha$ q promotes neurotransmission at the NMJ by activating two pathways through distinct downstream effectors: UNC-73/Trio RhoGEF and EGL-8/phospholipase C $\beta$ (PLC $\beta$ ) (Perez-Mansilla and Nurrish 2009). We found that either unc-73/Trio RhoGEF or egl-8/PLC $\beta$ putative null mutations partially block the effects of arecoline treatment on aldicarb sensitivity (Fig. 7D), suggesting that both pathways contribute to muscarinic signaling in motor neurons. However, unc-73/Trio RhoGEF but not egl-8/PLC $\beta$ mutants decreased SPHK-1-GFP punctal fluorescence compared with controls (Fig. 7A). Trio is a conserved guanine nucleotide exchange factor (GEF) family member that regulates axon guidance, synaptic growth, and neuronal function (Bateman and Van Vactor 2001; Williams et al. 2007; Ball et al. 2010). UNC73/Trio RhoGEF is an UNC-73 isoform that has a single Rho GTPase GEF domain that activates RHO-1/RhoA to promote NMJ function (McMullan et al. 2006; Williams et al. 2007). These results suggest that the unc-73/Trio RhoGEF branch of the egl-30/G $\alpha$ q signaling pathway mediates arecoline-dependent recruitment of SPHK-1 to synapses.

Our results show that $e g 1-8 / \mathrm{PLC} \beta$ does not positively regulate the abundance of SPHK-1-GFP at release sites. Consistent with this, mutations in signaling components acting downstream from EGL-8/PLC $\beta$, including PKC-1/ protein kinase $\mathrm{C}$ and DGK-1/diacylglycerol kinase, did not change SPHK-1-GFP punctal fluorescence compared with wild-type controls (Fig. 7A). Taken together, these data indicate that the EGL-30/G $\alpha$ q effector UNC-73/Trio but not the EGL-8/PLC $\beta$ or the DAG-PKC-1/PKC pathway positively regulates the abundance of SPHK-1-GFP at presynaptic terminals. Our data support a model (Fig. 7E) whereby ACh-activated $\mathrm{mAChR}-\mathrm{G} \alpha \mathrm{q}-$ Trio signaling recruits SPHK-1 to presynaptic release sites to positively modulate neurotransmitter release.

\section{Discussion}

Here we describe a new presynaptic component- the $C$. elegans SphK1 and SphK2 ortholog sphk-1-that promotes neurotransmitter release from motor terminals. SPHK-1 is concentrated at presynaptic membranes, where its abundance is regulated by endogenous ACh. ACh activates a conserved muscarinic signaling pathway in presynaptic motor neurons, consisting of a $\mathrm{mAChR}$, 
G $\alpha \mathrm{q} / 11$, and Trio RhoGEF, an effector of G $\alpha \mathrm{q}$. Our findings suggest that the regulation of SphK at sites of neurotransmitter release may be an important mechanism by which synaptic function is modulated by ACh. Furthermore, identifying regulators of sphingolipid metabolism enzymes is important, as misregulation of sphingolipids is implicated in neurological diseases such as Alzheimer's, Parkinson's, and Niemann Pick C (Okada et al. 2009).

\section{SPHK-1 positively regulates $A C h$ release}

Our data support the idea that SPHK-1 promotes ACh secretion. First, electrophysiological recordings show that sphk-1 mutants had reduced total charge transfer evoked by electrical stimulation. The amplitude and kinetics of spontaneous mEPSCs were similar to wild type, implying that muscle response to quantal release was not altered. Second, the accumulation of the SV markers SNB-1/ synaptobrevin and RAB-3/RAB3 in sphk-1 mutant synapses suggest a failure to efficiently release SVs from presynaptic terminals. Third, the aldicarb resistance and SV cycling defects of sphk-1 mutants were each rescued by presynaptic expression of $s p h k-1$. Taken together, these results strongly suggest that SPHK-1 potentiates synaptic transmission by increasing ACh release.

Interestingly, cultured mammalian neurons lacking the presynaptic calcium-binding protein DOC $2 \alpha$ or those overexpressing the SNARE-binding protein snapin exhibit a decreased total charge transfer defect (Pan et al. 2009; Yao et al. 2011), similar to that seen in sphk-1 mutants. The doc $2 \alpha$ mutant phenotype is due to impaired asynchronous neurotransmitter release, which represents the slower calcium-dependent component of evoked release. It is intriguing to speculate that SPHK-1 may be required during asynchronous release for the recruitment of reserve vesicles that contribute to sustained SV exocytosis. The accumulation of SVs at presynaptic terminals in $s p h k-1$ mutants may represent an accumulation of a pool of SVs that normally undergoes slow asynchronous release.

\section{Sphingolipid signaling and metabolism at synapses}

SPHK-1 is enriched at synapses and is likely to be associated with a region of the presynaptic plasma membrane near periactive zones for the following reasons. First, SPHK-1-GFP puncta occupied a small area within presynaptic terminals, as visualized by SNB-1-GFP. Second, SPHK-1-GFP remained punctate in unc-104/KIF1a mutants, in which SVs are no longer trafficked to axons. Third, mutants in which SV recycling is altered (unc-57/endophillin or unc-13/MUNC13 mutants) did not affect SPHK-1 abundance at presynaptic terminals. Finally, SPHK-1 colocalized most consistently but not completely with three different periactive zone markers, but less well with active zone proteins or dense core vesicles. Interestingly, mammalian SphK coimmunoprecipitates with the endocytosis proteins clathrin and Bin-1 (amphyphysin2) in murine mast cells (Urtz et al. 2004), and S1P binds the actin severing protein gelsolin (Bucki et al. 2010).
Our genetic evidence suggests that S1P but not SPH may promote SV secretion at motor terminals. First, sphk-1 transgenes that abolish its catalytic activity in motor neurons failed to rescue the aldicarb resistance and SV cycling defects of sphk-1 mutants, even though the protein still localized to presynaptic terminals. Second, hyl-1/CER synthase mutants, which have increased SPH levels (Menuz et al. 2009), displayed opposite locomotion phenotypes compared with sphk-1 mutants. Third, genetically increasing SPH levels while blocking generation of S1P (in hyl-1;sphk-1 double mutants) recapitulated the reduced locomotion defect of sphk-1 mutants. These results suggest that the neurotransmission defects associated with sphk-1 mutants cannot be accounted for by elevated SPH levels alone, but rather are likely due to reduced S1P generation. Consistent with this idea, sphk-1 mutants are more sensitive to radiation-induced germ cell apoptosis, a phenotype that is attributed to reduced S1P production (Deng et al. 2008). Our results are also consistent with other studies in which exogenous application of S1P increases neurotransmitter secretion in many cell types, including neurons (Brailoiu et al. 2002; Pan et al. 2006; Kajimoto et al. 2007), and the finding by Darios et al. (2009) that SPH mediates SNARE complex formation. This suggests that these two sphingolipids may both promote vesicle release and act via either distinct or codependent mechanisms.

$\mathrm{S} 1 \mathrm{P}$ is known to be either a secreted ligand that activates extracellular receptors (of the S1PR/EDG family) or an intracellular second messenger that targets cytoplasmic proteins (Spiegel 2000; Leclercq and Pitson 2006; Rosen et al. 2009). We found that sphk-1 acts both cell-autonomously in motor neurons and non-cell-autonomously in muscles to regulate aldicarb responsiveness and SV cycling. However, the C. elegans genome does not encode obvious S1PR/EDG family orthologs. Thus, we speculate that S1P generated in neurons acts as an intracellular messenger on synaptic targets within motor neurons to promote release. In addition, S1P generated in muscles may be able to diffuse to motor neurons, but likely acts by a mechanism independent of known S1P receptors. S1P can interact with multiple intracellular targets, including transient receptor potential (TRP) channels, store-operated calcium channels, inositol triphosphate receptors, and calcium-activated potassium channels $\left(\mathrm{BK}_{\mathrm{Ca}}\right.$ ) (Ghosh et al. 1994; Mattie et al. 1994; Flemming et al. 2006; Kim et al. 2006). Although these targets are found in neurons, the functional significance of their interaction with S1P on neurotransmitter release is not known. Recently, intracellular S1P has also been shown to directly associate with and modulate the activities of the E3 ubiquitin ligase TRAF2 and the histone deacetylases (HDAC1 and HDAC2) (Hait et al. 2009; Alvarez et al. 2010). HDAC2 regulates synaptic plasticity and memory formation in mouse models (Guan et al. 2009), raising the possibility that regulation of histone deacetylase activity by S1P may contribute to plasticity.

\section{Muscarinic regulation of SPHK-1 synaptic abundance}

We found that experimentally manipulating levels of SPHK-1 at synapses led to corresponding changes in 
aldicarb responses. Overexpressing SPHK-1 resulted in increased SPHK-1 abundance at synapses and conferred aldicarb hypersensitivity to animals. In contrast, reducing SPHK-1 abundance by $\sim 50 \%$ without reducing catalytic activity (by deleting the CaM-binding domain of SPHK-1) abolished its ability to rescue the aldicarb resistance of sphk-1 mutants. Together, these observations suggest that presynaptic levels of SPHK-1 may be critical for its function in synaptic transmission.

ACh was necessary and sufficient for SPHK-1 synaptic recruitment. Treatment of wild-type animals with arecoline resulted in an $\sim 30 \%$ increase in SPHK-1 punctal fluorescence, whereas reduction of endogenously produced ACh (in cha-1/ChAT temperature-sensitive mutants) resulted in an $\sim 30 \%$ decrease in SPHK-1-GFP punctal fluorescence. These changes are not secondary consequences of altering cholinergic transmission because mutations that either increase or decrease ACh secretion (in $c p x-1$ ) complexin or unc-13/Munc13 mutants, respectively) did not change SPHK-1 abundance. Thus, the effects of ACh on SPHK-1 abundance reflect the specific recruitment of SPHK-1 by ACh signaling. The effects of ACh and arecoline on SPHK-1 recruitment were relatively rapid: We observed a decrease in punctal fluorescence within 15 min of temperature shift of cha-1/ChAT mutants. These results are consistent with the established notion that SphK membrane abundance is regulated by its recruitment to membranes from cytoplasmic pools (Pitson et al. 2003). However, we cannot rule out other mechanisms that might regulate synaptic SPHK-1 abundance at synapses, such as changes in the stability of synaptic SPHK-1 or its diffusion within axonal membranes. Importantly, the effect of arecoline on aldicarb responsiveness was dramatically reduced by sphk-1 mutations, indicating that SPHK-1 activity is required, at least in part, for the effects of muscarinic signaling on synaptic transmission.

A previous study has shown that depolarization of cultured neurons recruits SphK to axonal membranes (Kajimoto et al. 2007). Consistent with this, we found that presynaptic calcium may regulate SPHK-1 synaptic recruitment, since removing the CaM-binding domain of SPHK-1 reduced its synaptic abundance. In addition, reducing presynaptic activity [in slo-1(gf)/BK channel mutants] also reduced SPHK-1-GFP synaptic localization. Therefore, depolarizing presynaptic terminals is important for SPHK-1 synaptic recruitment, but whether it is required for muscarinic-mediated regulation of SPHK-1 remains unclear.

\section{Trio RhoGEF regulates SPHK-1 abundance}

We found that the M1/M3/M5 class of mAChR, gar-3, acts cell-autonomously in motor neurons to mediate the effects of arecoline on SPHK-1 abundance at presynaptic terminals. In mammals, activation of the M1 and M3 classes of $\mathrm{mAChRs}$ results in activation of $\mathrm{G} \alpha \mathrm{q}$, which in turn primarily activates PLC. Although we found that EGL-8/PLC $\beta$ mediates some of the effects of muscarinic agonists at synapses, our data do not support a role for EGL-8/PLC $\beta$ in recruiting SPHK-1 to release sites, since egl-8/PLC $\beta$ mutations (or mutations in $d g k-1 /$ diacylglycerol kinase or $p k c-1 /$ protein kinase C) did not reduce SPHK-1GFP punctal fluorescence. Interestingly, SPHK-1-GFP fluorescence increased in egl-8/PLC $\beta$ mutants, suggesting that egl-8/PLC $\beta$ may negatively regulate SPHK-1 localization. PLC $\beta$ has G $\alpha \mathrm{q}$ GAP activity (Ross 2011), suggesting that EGL-30/Gaq may be able to activate UNC-73/Trio in egl-8/PLC $\beta$ mutants. Alternatively, EGL8/PLC $\beta$ may compete with UNC-73/Trio for activation by EGL-30/G $\alpha$ q, which may lead to an enhancement of UNC$73 /$ Trio signaling in the absence of egl-8/PLC $\beta$.

We identified an alternative G $\alpha \mathrm{q}$ effector, unc-73/Trio, that acts downstream from $\mathrm{mAChR}$ and $e g 1-30 / \mathrm{G} \alpha \mathrm{q}$ to regulate SPHK-1 abundance. Trios comprise a large family of proteins with diverse functions that act by regulating GTPases of the Rac and Rho family. Of note, Kalirin 7, a Trio family member, is required for synaptic function of hippocampal neurons and for peptide secretion (Mains et al. 1999; Ma et al. 2008). In C. elegans, the unc-73/Trio locus encodes six isoforms, which differ in the number of GEF domains and their tissue distributions. Our results show that a mutation that specifically disrupts the RhoGEF domain of unc-73, which is found within the neuronally expressed UNC-73E isoform (Steven et al. 2005), reduces SPHK-1 at presynaptic terminals. Rho GTPase activity is implicated in recruiting UNC-13/ Munc13 to synapses (McMullan et al. 2006), and specific loss of Trio's Rho GTPase activity decreases neuromuscular function (Steven et al. 2005; Williams et al. 2007). Interestingly, M3 mAChR stimulation has been shown to activate p114-RhoGEF (Blomquist et al. 2000; Rumenapp et al. 2001) and M1 mAChR to activate leukemia-associated RhoGEF (LARG) (Booden et al. 2002). Thus, utilization of RhoGEFs may be a universal mechanism by which cholinergic signaling regulates cellular function. However, other pathways in addition to unc-73/Trio are likely to contribute to the synaptic localization of SPHK-1, since mutations in egl-30/G $\alpha$ q or unc-73/Trio components reduced, but did not eliminate, synaptic abundance of SPHK-1-GFP.

Our results point to a model in which the muscarinic signaling pathway consisting of GAR-3/mAChR, EGL30/Gaq, and UNC-73/Trio recruits SPHK-1 to presynaptic membranes near sites of vesicle release. SPHK-1 recruitment leads to local production of $\mathrm{S} 1 \mathrm{P}$, which in turn binds to one or more unidentified synaptic targets to promote neurotransmitter secretion. By regulating relative levels of SPH and S1P near periactive zones, SPHK-1 may be involved in coupling exocytosis and endocytosis during activity-modulated synaptic transmission. It will be important to identify the endogenous source of $\mathrm{ACh}$ that promotes SPHK-1 synaptic recruitment as well as the synaptic targets of S1P that mediate the effects of ACh on SV release.

\section{Materials and methods}

\section{C. elegans strains}

Strains sphk-1(ok1097) and hyl-1(gk203) were provided by the Caenorhabditis Genetics Center, which is funded by the NIH National Center for Research Resources (NCRR). The wild-type reference strain was N2 Bristol. sphk-1(ok1097) was outcrossed 
10 times and used for all experiments. All other strains used were outcrossed more than four times. In addition, the following strains were used (strain information is at http://www.wormbase.org): cha-1(p1182), cpx-1(ok1522), dgk-1(nu62), egl-8(sa47), egl-30(ad806), egl-30(js126gf), gar-1(ok755), gar-3(gk305), pkc-1(nj3), slo-1(ky399gf), tomo-1(nu468), unc-13(s69), unc-18(md299), unc-57(e406), unc73(ce362), unc-104(e1265), nuIs152[ttx-3-mRFP, Punc-129GFP-snb-1]II, nuIs159[ttx-3-mRFP, Punc-129-GFP-syd-2]III, nuIs162[myo-2-GFP, Punc-129-snn-1-Venus]III, nuIs166[myo2-GFP, Punc-129-unc-10:GFP]III, nuIs168[myo-2-GFP, Punc-129Venus-rab-3]IV, nuIs169[myo-2-GFP, Punc-129-gelsolin-Venus]III, nuIs175[myo-2-RFP, Punc-129-RFP-snb-1]X, nuIs183[myo-2-GFP, Punc-129-nlp-21-Venus]III, nuIs184[myo-2-GFP, Punc-129-apt-4GFP]X, nuIs197[myo-2-GFP, Punc-129-sphk-1-GFP]III, nuIs214 [myo-2-GFP, Punc-129-itsn-1-GFP]III, and nuIs321[myo-2-GFP, Punc-17-RFP]. All integrated transgenes were outcrossed 10 times.

\section{Molecular biology}

The promoter fragment of $s p h k-1$ was amplified by PCR from mixed-stage genomic DNA. sphk-1 mutations were made by QuikChange PCR (Stratagene) targeting the following deletions: kinase-dead (KD1) (S101A, G102A, D103A, G104A, and L105A), kinase-dead (KD2) (G184D, S185D, G185D, and N186D), and $\triangle \mathrm{CaM}$ (F271A and I272Q). For a full list of primers used, see the Supplemental Material. The cDNA was used to make the following plasmids by standard molecular biology techniques (plasmid name[promoter-gene-marker]): pDS221[Punc-129-sphk1-GFP], pDS300[Psphk-1-GFP-NLS], pDS301[Punc-129-sphk-1mCherry], pDS302[Pmyo-3-sphk-1-GFP], pDS303[Psphk-1-sphk1-GFP], pDS304[Psnb-1-sphk-1-GFP], pDS305[Punc-129-sphk-1], pDS306[Punc-129-sphk-1(CaM)-GFP], pDS307[Punc-129-sphk1(KD)], pDS308[Pmyo-3-sphk-1], pDS309[Psphk-1-sphk-1(KD)-GFP], pDS323[Punc-129-gar-3], and pDS361[Psnb-1-sphk-1(CaM)-GFP].

\section{Transgenic lines}

Transgenic strains were generated by injecting either N2, sphk1(ok1097), sphk-1(ok1097);nuIs152, or nuIs197 animals with expression constructs $(10-25 \mathrm{ng} / \mu \mathrm{L})$ and the coinjection marker KP\#708 (Pttx-3-rfp, 40 ng/ $\mu \mathrm{L}$ ) or KP\#1 106 (Pmyo-2-gfp 10 ng/ $\mu \mathrm{L}$ ). Microinjection was performed using standard techniques as previously described (Mello et al. 1991). At least three lines for each transgene were examined, and either an average of the three transgenes or results from a single, representative transgene are shown. For transgene information, see the Supplemental Material.

Behavioral analysis of locomotion activity and sensitivity to aldicarb

For analysis of locomotion activity, the number of body bends per minute was counted for L4 worms on normal growth medium (NGM) plates in the presence of food, as previously described (McMullan et al. 2006). Thirty worms were scored for each group. For analysis of sensitivity to inhibitors of acetylcholinesterase, paralysis of adult worms was scored every $10 \mathrm{~min}$, starting at 40 min, using 0.5-2 mM aldicarb (Chem Services). For each experiment, three plates of 20 worms per genotype were placed on NGM plates supplemented with aldicarb, and the number of worms paralyzed on each plate was counted to extract the percent paralyzed at each time point per genotype. The percentages were averaged at each time point per genotype and plotted graphically. For each experiment, the genotype was blind to the scorer, and the analysis was repeated at least two times. Statistical differences were determined at each time point by Student's $t$-tests.

\section{Electrophysiology}

Electrophysiology was done on dissected C. elegans as described (Richmond and Jorgensen 1999). Worms were superfused in an extracellular solution containing $127 \mathrm{mM} \mathrm{NaCl}, 5 \mathrm{mM} \mathrm{KCl}, 26$ $\mathrm{mM} \mathrm{NaHCO} 3,1.25 \mathrm{mM} \mathrm{NaH} \mathrm{PO}_{4}, 20 \mathrm{mM}$ glucose, $3 \mathrm{mM}$ $\mathrm{CaCl}_{2}$, and $3 \mathrm{mM} \mathrm{MgCl}$ (330 mOsm at $\left.\mathrm{pH} 7.2\right)$, bubbled with $5 \% \mathrm{CO}_{2}$ and $95 \% \mathrm{O}_{2}$ at $20^{\circ} \mathrm{C}$. Whole-cell recordings were carried out at $-60 \mathrm{mV}$ using an internal solution containing $105 \mathrm{mM}$ $\mathrm{CH}_{3} \mathrm{O}_{3} \mathrm{SCs}, 10 \mathrm{mM} \mathrm{CsCl}, 15 \mathrm{mM}$ CsF, $4 \mathrm{mM} \mathrm{MgCl}_{2}, 5 \mathrm{mM}$ EGTA, $0.25 \mathrm{mM} \mathrm{CaCl}_{2}, 10 \mathrm{mM} \mathrm{HEPES}$, and $4 \mathrm{mM} \mathrm{Na}_{2} \mathrm{ATP}$ (315 mOsm, adjusted to $\mathrm{pH} 7.2$ using $\mathrm{CsOH}$ ). Under these conditions, we only observed endogenous ACh EPSCs. All other recording conditions were as described (Sieburth et al. 2007). Statistical significance was determined by Student's $t$-test.

\section{Microscopy and analysis}

Fluorescence microscopy experiments were performed as previously described (Ch'ng et al. 2008). Briefly, adult worms were paralyzed using 2,3-butanedione monoxime (BDM, $30 \mu \mathrm{g} / \mu \mathrm{L}$; Sigma) and mounted on $2 \%$ agarose pads for imaging. Images of synapses were captured from dorsal axons of DA neurons near the posterior gonadal bend of the worm. Approximately 30 worms (spanning 500 synapses) were imaged for all experiments. For all fluorescence microscopy experiments, images were captured with a Nikon eclipse 90i microscope equipped with a Nikon PlanApo $100 \times$ objective $(\mathrm{NA}=1.4)$ and a Photometrics Coolsnap ES ${ }^{2}$ camera. Metamorph 7.0 software (Universal Imaging/Molecular Devices) was used to capture serial image stacks, and the maximum intensity projection was used for analysis of the dorsal cords. Line scans of the maximum intensity projection image were also recorded using Metamorph. The fluorescence intensity values were then quantified using Puncta 6.0 software written with Igor Pro (Wavemetrics), as previously described (Dittman and Kaplan 2006; Ch'ng et al. 2008). For all experiments, fluorescence values were normalized to the values of $0.5 \mu \mathrm{m}$ FluoSphere beads (Invitrogen) captured during each imaging session. This was performed to provide a standard for comparing absolute fluorescence levels between animals from different sessions.

We found that the conditions used for imaging showed that SPHK-1-GFP, UNC-10-GFP, and GFP-SYD-2 were predominantly localized to synaptic puncta, as determined previously $\left(\mathrm{Ch}^{\prime} \mathrm{ng}\right.$ et al. 2008). There was little or no difference between their axonal fluorescence and the autofluorescence observed at C. elegans axons. Therefore, we excluded the axonal fluorescence in our analysis for these markers (see Fig. 2; Supplemental Tables S2, S3). In addition, we performed analyses of SPHK-1 localization with either SPHK-1GFP or SPHK-1-mCherry fusion proteins paired with other fluorescently tagged synaptic markers because the fusion proteins SPHK-1-GFP and SPHK-1-mCherry showed similar localization patterns (Supplemental Fig. S2) when colocalized in the same cell.

\section{Pharmacology}

Adult animals were pretreated for $2 \mathrm{~h}$ on NGM plates supplemented with arecoline (15 mM in M9; Sigma), serotonin (10 mM) or SKI (50 $\mu \mathrm{M}$ in DMSO; EMD Chemicals). For controls, NMG plates were supplemented with a control solvent (M9, DMSO, or water) for the respective experiments. For aldicarb experiments, drugs were also added to the aldicarb plates.

\section{Statistical analysis}

For all experiments comparing two samples, a Student's $t$-test was used to determine significance, set at $P<0.05$. For experiments 
comparing multiple groups, significance was determined by performing a one-way ANOVA and a Tukey post-hoc analysis. Propagation of standard error of the mean (SEM) was performed for all comparisons. Significance was also set at $P<0.05$. Full charts of statistical analyses for all experimental comparisons are available in the Supplemental Material (Supplemental Tables S1-S3).

\section{Acknowledgments}

We thank the following individuals for contributions and advice: J. Kaplan, Q. Ch'ng, J. Chen, T. Staab, H. Wang, and M. Olahova for critical reading of the manuscript; B. Ramos for laboratory reagents; S. Gorda for technical assistance; and Q. Ch'ng and J. Dittman for fluorescence analysis software. This work was funded by grants from the American Heart Association and NIH NINDS (NS071085-02). The Caenorhabditis Genetics Center provided the strains used in this study.

\section{References}

Alemany R, Kleuser B, Ruwisch L, Danneberg K, Lass H, Hashemi R, Spiegel S, Jakobs KH, Meyer zu Heringdorf D. 2001. Depolarisation induces rapid and transient formation of intracellular sphingosine-1-phosphate. FEBS Lett 509: 239-244.

Alemany R, van Koppen CJ, Danneberg K, Ter Braak M, Meyer $\mathrm{Zu}$ Heringdorf D. 2007. Regulation and functional roles of sphingosine kinases. Naunyn Schmiedebergs Arch Pharmacol 374: 413-428.

Alvarez SE, Harikumar KB, Hait NC, Allegood J, Strub GM, Kim EY, Maceyka M, Jiang H, Luo C, Kordula T, et al. 2010. Sphingosine-1-phosphate is a missing cofactor for the E3 ubiquitin ligase TRAF2. Nature 465: 1084-1088.

Ball RW, Warren-Paquin M, Tsurudome K, Liao EH, Elazzouzi F, Cavanagh C, An B-S, Wang T-T, White JH, Haghighi AP. 2010. Retrograde BMP signaling controls synaptic growth at the NMJ by regulating trio expression in motor neurons. Neuron 66: 536-549.

Bateman J, Van Vactor D. 2001. The Trio family of guaninenucleotide-exchange factors: Regulators of axon guidance. J Cell Sci 114: 1973-1980.

Blomquist A, Schworer G, Schablowski H, Psoma A, Lehnen M, Jakobs KH, Rumenapp U. 2000. Identification and characterization of a novel Rho-specific guanine nucleotide exchange factor. Biochem I 352: 319-325.

Booden MA, Siderovski DP, Der CJ. 2002. Leukemia-associated Rho guanine nucleotide exchange factor promotes G $\alpha \mathrm{q}-$ coupled activation of RhoA. Mol Cell Biol 22: 4053-4061.

Brailoiu E, Cooper RL, Dun NJ. 2002. Sphingosine 1-phosphate enhances spontaneous transmitter release at the frog neuromuscular junction. Br J Pharmacol 136: 1093-1097.

Brown DA. 2010. Muscarinic acetylcholine receptors (mAChRs) in the nervous system: Some functions and mechanisms. J Mol Neurosci 41: 340-346.

Bryan L, Kordula T, Spiegel S, Milstien S. 2008. Regulation and functions of sphingosine kinases in the brain. Biochim Biophys Acta 1781: 459-466.

Bucki R, Kulakowska A, Byfield FJ, Zendzian-Piotrowska M, Baranowski M, Marzec M, Winer JP, Ciccarelli NJ, Górski J, Drozdowski W, et al. 2010. Plasma gelsolin modulates cellular response to sphingosine 1-phosphate. Am I Physiol Cell Physiol 299: C1516-C1523. doi: 10.1152/ajpcell. 00051.2010

Burns AR, Wallace IM, Wildenhain J, Tyers M, Giaever G, Bader GD, Nislow C, Cutler SR, Roy PJ. 2010. A predictive model for drug bioaccumulation and bioactivity in Caenorhabditis elegans. Nat Chem Biol 6: 549-557.
Camoletto PG, Vara H, Morando L, Connell E, Marletto FP, Giustetto M, Sassoè-Pognetto M, Van Veldhoven PP, Ledesma MD. 2009. Synaptic vesicle docking: Sphingosine regulates syntaxin1 interaction with Munc18. PLOS ONE 4: e5310. doi: 10.1371/journal.pone.0005310.

Ch'ng Q, Sieburth D, Kaplan JM. 2008. Profiling synaptic proteins identifies regulators of insulin secretion and lifespan. PLoS Genet 4: e1000283. doi: 10.1371/journal.pgen.1000283.

Darios F, Wasser C, Shakirzyanova A, Giniatullin A, Goodman K, Munoz-Bravo JL, Raingo J, Jorgačevski J, Kreft M, Zorec R, et al. 2009. Sphingosine facilitates SNARE complex assembly and activates synaptic vesicle exocytosis. Neuron 62: 683-694.

Deng X, Yin X, Allan R, Lu DD, Maurer CW, HaimovitzFriedman A, Fuks Z, Shaham S, Koslenick R. 2008. Ceramide biogenesis is required for radiation-induced apoptosis in the germ line of C. elegans. Science 322: 110-115.

Dittman JS, Kaplan JM. 2006. Factors regulating the abundance and localization of synaptobrevin in the plasma membrane. Proc Natl Acad Sci 103: 11399-11404.

Drever BD, Riedel G, Platt B. 2011. The cholinergic system and hippocampal plasticity. Behav Brain Res 221: 505-514.

Fernandez-Alfonso T, Kwan R, Ryan TA. 2006. Synaptic vesicles interchange their membrane proteins with a large surface reservoir during recycling. Neuron 51: 179-186.

Flemming PK, Dedman AM, Xu SZ, Li J, Zeng F, Naylor J, Benham CD, Bateson AN, Muraki K, Beech DJ. 2006. Sensing of lysophospholipids by TRPC5 calcium channel. J Biol Chem 281: 4977-4982.

Fox RM, Von Stetina SE, Barlow SJ, Shaffer C, Olszewski KL, Moore JH, Dupuy D, Vidal M, Miller DM III. 2005. A gene expression fingerprint of C. elegans embryonic motor neurons. BMC Genomics 6: 42. doi: 10.1186/1471-2164-6-42.

Ghosh TK, Bian J, Gill DL. 1994. Sphingosine 1-phosphate generated in the endoplasmic reticulum membrane activates release of stored calcium. I Biol Chem 269: 22628-22635.

Gracheva EO, Burdina AO, Holgado AM, Berthelot-Grosjean M, Ackley BD, Hadwiger G, Nonet ML, Weimer RM, Richmond JE. 2006. Tomosyn inhibits synaptic vesicle priming in Caenorhabditis elegans. PLoS Biol 4: e261. doi: 10.1371/ journal.pbio.0040261.

Guan JS, Haggarty SJ, Giacometti E, Dannenberg JH, Joseph N, Gao J, Nieland TJF, Zhou Y, Wang X, Mazitschek R, et al. 2009. HDAC2 negatively regulates memory formation and synaptic plasticity. Nature 459: 55-60.

Hait NC, Allegood J, Maceyka M, Strub GM, Harikumar KB, Singh SK, Luo C, Marmorstein R, Kordula T, Milstien S, et al. 2009. Regulation of histone acetylation in the nucleus by sphingosine-1-phosphate. Science 325: 1254-1257.

Hall DH, Hedgecock EM. 1991. Kinesin-related gene unc-104 is required for axonal transport of synaptic vesicles in $C$. elegans. Cell 65: 837-847.

Hannun YA, Obeid LM. 2008. Principles of bioactive lipid signalling: Lessons from sphingolipids. Nat Rev Mol Cell Biol 9: 139-150.

Hobson RJ, Liu Q, Watanabe S, Jorgensen EM. 2011. Complexin maintains vesicles in the primed state in C. elegans. Curr Biol 21: 106-113.

Jarman KE, Moretti PA, Zebol JR, Pitson SM. 2010. Translocation of sphingosine kinase 1 to the plasma membrane is mediated by calcium- and integrin-binding protein 1. J Biol Chem 285: 483-492.

Kage-Nakadai E, Kobuna H, Kimura M, Gengyo-Ando K, Inoue T, Arai H, Mitani S. 2010. Two very long chain fatty acid acyl-CoA synthetase genes, acs-20 and acs-22, have roles in the cuticle surface barrier in Caenorhabditis elegans. PLoS ONE 5: e8857. doi: 10.1371/journal.pone.0008857. 
Kajimoto T, Okada T, Yu H, Goparaju SK, Jahangeer S, Nakamura S. 2007. Involvement of sphingosine-1-phosphate in glutamate secretion in hippocampal neurons. Mol Cell Biol 27: 3429-3440.

Kanno T, Nishizaki T, Proia RL, Kajimoto T, Jahangeer S, Okada T, Nakamura S. 2010. Regulation of synaptic strength by sphingosine 1-phosphate in the hippocampus. Neuroscience 171: 973-980.

Kim MY, Liang GH, Kim JA, Kim YJ, Oh S, Suh SH. 2006. Sphingosine-1-phosphate activates BKCa channels independently of G protein-coupled receptor in human endothelial cells. Am I Physiol Cell Physiol 290: C1000-C1008. doi: 10.1152/ajpcell.00353.2005.

Lackner MR, Nurrish SJ, Kaplan JM. 1999. Facilitation of synaptic transmission by EGL-30 Gq $\alpha$ and EGL-8 PLC $\beta$ : DAG binding to UNC-13 is required to stimulate acetylcholine release. Neuron 24: 335-346.

Leclercq TM, Pitson SM. 2006. Cellular signalling by sphingosine kinase and sphingosine 1-phosphate. IUBMB Life 58: 467-472.

Liu Q, Chen B, Ge Q, Wang ZW. 2007. Presynaptic $\mathrm{Ca}^{2+} /$ calmodulin-dependent protein kinase II modulates neurotransmitter release by activating BK channels at Caenorhabditis elegans neuromuscular junction. J Neurosci 27: 10404-10413.

Liu Y, LeBoeuf B, Garcia LR. 2007. G $\alpha$ (q)-coupled muscarinic acetylcholine receptors enhance nicotinic acetylcholine receptor signaling in Caenorhabditis elegans mating behavior. I Neurosci 27: 1411-1421.

Ma XM, Kiraly DD, Gaier ED, Wang Y, Kim EJ, Levine ES, Eipper BA, Mains RE. 2008. Kalirin-7 is required for synaptic structure and function. J Neurosci 28: 12368-12382.

Madison JM, Nurrish S, Kaplan JM. 2005. UNC-13 interaction with syntaxin is required for synaptic transmission. Curr Biol 15: 2236-2242.

Mains RE, Alam MR, Johnson RC, Darlington DN, Back N, Hand TA, Eipper BA. 1999. Kalirin, a multifunctional PAM $\mathrm{COOH}$-terminal domain interactor protein, affects cytoskeletal organization and ACTH secretion from AtT-20 cells. I Biol Chem 274: 2929-2937.

Martin JA, Hu Z, Fenz KM, Fernandez J, Dittman JS. 2011. Complexin has opposite effects on two modes of synaptic vesicle fusion. Curr Biol 21: 97-105.

Mattie M, Brooker G, Spiegel S. 1994. Sphingosine-1-phosphate, a putative second messenger, mobilizes calcium from internal stores via an inositol trisphosphate-independent pathway. I Biol Chem 269: 3181-3188.

McMullan R, Hiley E, Morrison P, Nurrish SJ. 2006. Rho is a presynaptic activator of neurotransmitter release at preexisting synapses in C. elegans. Genes Dev 20: 65-76.

Mello CC, Kramer JM, Stinchcomb D, Ambros V. 1991. Efficient gene transfer in C. elegans: Extrachromosomal maintenance and integration of transforming sequences. EMBO J 10: 39593970.

Menuz V, Howell KS, Gentina S, Epstein S, Riezman I, FornallazMulhauser M, Hengartner MO, Gomez M, Riezman H, Martinou JC. 2009. Protection of C. elegans from anoxia by HYL-2 ceramide synthase. Science 324: 381-384.

Meyer zu Heringdorf D, Lass H, Alemany R, Laser KT, Neumann E, Zhang C, Schmidt M, Rauen U, Jakobs KH, van Koppen CJ. 1998. Sphingosine kinase-mediated $\mathrm{Ca}^{2+}$ signalling by G-protein-coupled receptors. EMBO I 17: 2830-2837.

Mizugishi K, Yamashita T, Olivera A, Miller GF, Spiegel S, Proia RL. 2005. Essential role for sphingosine kinases in neural and vascular development. Mol Cell Biol 25: 11113-11121.

Nicol GD. 2008. Nerve growth factor, sphingomyelins, and sensitization in sensory neurons. Sheng Li Xue Bao 60: 603-604.
Nurrish S, Segalat L, Kaplan JM. 1999. Serotonin inhibition of synaptic transmission: $\mathrm{G} \alpha(0)$ decreases the abundance of UNC-13 at release sites. Neuron 24: 231-242.

Okada T, Kajimoto T, Jahangeer S, Nakamura SI. 2009. Sphingosine kinase/sphingosine 1-phosphate signalling in central nervous system. Cell Signal 21: 7-13.

Olivera A. 2008. Unraveling the complexities of sphingosine1-phosphate function: The mast cell model. Prostaglandins Other Lipid Mediat 86: 1-11.

Olivera A, Spiegel S. 1993. Sphingosine-1-phosphate as second messenger in cell proliferation induced by PDGF and FCS mitogens. Nature 365: 557-560.

Ou CY, Shen K. 2011. Neuronal polarity in C. elegans. Dev Neurobiol 71: 554-566.

Pan CY, Lee H, Chen CL. 2006. Lysophospholipids elevate $\left[\mathrm{Ca}^{2+}\right]_{i}$ and trigger exocytosis in bovine chromaffin cells. Neuropharmacology 51: 18-26.

Pan PY, Tian JH, Sheng ZH. 2009. Snapin facilitates the synchronization of synaptic vesicle fusion. Neuron 61: 412-424.

Perez-Mansilla B, Nurrish S. 2009. A network of G-protein signaling pathways control neuronal activity in C. elegans. Adv Genet 65: 145-192.

Pfaff M, Powaga N, Akinci S, Schütz W, Banno Y, Wiegand S, Kummer W, Wess J, Haberberger RV. 2005. Activation of the SPHK/S1P signalling pathway is coupled to muscarinic receptor-dependent regulation of peripheral airways. Respir Res 6: 48. doi: 10.1186/1465-9921-6-48.

Pitson SM. 2011. Regulation of sphingosine kinase and sphingolipid signaling. Trends Biochem Sci 36: 97-107.

Pitson SM, Moretti PA, Zebol JR, Zareie R, Derian CK, Darrow AL, Qi J, D'Andrea RJ, Bagley CJ, Vadas MA, et al. 2002. The nucleotide-binding site of human sphingosine kinase 1. I Biol Chem 277: 49545-49553.

Pitson SM, Moretti PA, Zebol JR, Lynn HE, Xia P, Vadas MA, Wattenberg BW. 2003. Activation of sphingosine kinase 1 by ERK1/2-mediated phosphorylation. EMBO J 22: 5491-5500.

Rand JB. 1989. Genetic analysis of the cha-1-unc-17 gene complex in Caenorhabditis. Genetics 122: 73-80.

Richmond JE, Jorgensen EM. 1999. One GABA and two acetylcholine receptors function at the C. elegans neuromuscular junction. Nat Neurosci 2: 791-797.

Richmond JE, Davis WS, Jorgensen EM. 1999. UNC-13 is required for synaptic vesicle fusion in C. elegans. Nat Neurosci 2: 959-964.

Richmond JE, Weimer RM, Jorgensen EM. 2001. An open form of syntaxin bypasses the requirement for UNC-13 in vesicle priming. Nature 412: 338-341.

Rohrbough J, Rushton E, Palanker L, Woodruff E, Matthies HJ, Acharya U, Acharya JK, Broadie K. 2004. Ceramidase regulates synaptic vesicle exocytosis and trafficking. I Neurosci 24: 7789-7803.

Rosen H, Gonzalez-Cabrera PJ, Sanna MG, Brown S. 2009. Sphingosine 1-phosphate receptor signaling. Annu Rev Biochem 78: 743-768.

Ross EM. 2011. G $\alpha \mathrm{q}$ and phospholipase C- $\beta$ : Turn on, turn off, and do it fast. Sci Signal 4: pe5. doi: 10.1126/scisignal. 2001798.

Rumenapp U, Asmus M, Schablowski H, Woznicki M, Han L, Jakobs KH, Fahimi-Vahid M, Michalek C, Wieland T, Schmidt M. 2001. The M3 muscarinic acetylcholine receptor expressed in HEK-293 cells signals to phospholipase D via G12 but not Gq-type $G$ proteins: Regulators of $G$ proteins as tools to dissect pertussis toxin-resistant $G$ proteins in receptor-effector coupling. J Biol Chem 276: 2474-2479.

Sarkar S, Maceyka M, Hait NC, Paugh SW, Sankala H, Milstien S, Spiegel S. 2005. Sphingosine kinase 1 is required for 
migration, proliferation and survival of MCF-7 human breast cancer cells. FEBS Lett 579: 5313-5317.

Sieburth D, Ch'ng Q, Dybbs M, Tavazoie M, Kennedy S, Wang D, Dupuy D, Rual J-F, Hill DE, Vidal M, et al. 2005. Systematic analysis of genes required for synapse structure and function. Nature 436: 510-517.

Sieburth D, Madison JM, Kaplan JM. 2007. PKC-1 regulates secretion of neuropeptides. Nat Neurosci 10: 49-57.

Spiegel S. 2000. Sphingosine 1-phosphate: A ligand for the EDG-1 family of G-protein-coupled receptors. Ann N Y Acad Sci 905: 54-60.

Spiegel S, Cuvillier O, Edsall LC, Kohama T, Menzeleev R, Olah Z, Olivera A, Pirianov G, Thomas DM, Tu Z, et al. 1998. Sphingosine-1-phosphate in cell growth and cell death. Ann N Y Acad Sci 845: 11-18.

Stahelin RV, Hwang JH, Kim JH, Park ZY, Johnson KR, Obeld LM, Cho W. 2005. The mechanism of membrane targeting of human sphingosine kinase 1. J Biol Chem 280: 43030-43038.

Steger KA, Avery L. 2004. The GAR-3 muscarinic receptor cooperates with calcium signals to regulate muscle contraction in the Caenorhabditis elegans pharynx. Genetics 167: 633-643.

Steven R, Zhang L, Culotti J, Pawson T. 2005. The UNC-73/Trio RhoGEF-2 domain is required in separate isoforms for the regulation of pharynx pumping and normal neurotransmission in C. elegans. Genes Dev 19: 2016-2029.

Sutherland CM, Moretti PA, Hewitt NM, Bagley CJ, Vadas MA, Pitson SM. 2006. The calmodulin-binding site of sphingosine kinase and its role in agonist-dependent translocation of sphingosine kinase 1 to the plasma membrane. J Biol Chem 281: 11693-11701.

ter Braak M, Danneberg K, Lichte K, Liphardt K, Ktistakis NT, Pitson SM, Hla T, Jakobs KH, Meyer zu Heringdorf D. 2009. $\mathrm{G} \alpha(\mathrm{q})$-mediated plasma membrane translocation of sphingosine kinase-1 and cross-activation of S1P receptors. Biochim Biophys Acta 1791: 357-370.

Urtz N, Olivera A, Bofill-Cardona E, Csonga R, Billich A, Mechtcheriakova D, Bornancin F, Woisetschläger M, Rivera J, Baumruker T. 2004. Early activation of sphingosine kinase in mast cells and recruitment to FceRI are mediated by its interaction with Lyn kinase. Mol Cell Biol 24: 8765-8777.

Vashlishan AB, Madison JM, Dybbs M, Bai J, Sieburth D, Ch'ng Q, Tavazoie M, Kaplan JM. 2008. An RNAi screen identifies genes that regulate GABA synapses. Neuron 58: 346-361.

Weimer RM, Richmond JE, Davis WS, Hadwiger G, Nonet ML, Jorgensen EM. 2003. Defects in synaptic vesicle docking in unc-18 mutants. Nat Neurosci 6: 1023-1030.

Williams SL, Lutz S, Charlie NK, Vettel C, Ailion M, Coco C, Tesmer JJG, Jorgensen EM, Wieland T, Miller KG. 2007. Trio's Rho-specific GEF domain is the missing G $\alpha$ q effector in C. elegans. Genes Dev 21: 2731-2746.

Yao J, Gaffaney JD, Kwon SE, Chapman ER. 2011. Doc2 is a Ca ${ }^{2+}$ sensor required for asynchronous neurotransmitter release. Cell 147: 666-677.

Yonamine I, Bamba T, Nirala NK, Jesmin N, KosakowskaCholody T, Nagashima K, Fukusaki E, Acharya JK, Acharya U. 2011. Sphingosine kinases and their metabolites modulate endolysosomal trafficking in photoreceptors. I Cell Biol 192: $557-567$.

Young KW, Willets JM, Parkinson MJ, Bartlett P, Spiegel S, Nahorski SR, Challiss RAJ. 2003. $\mathrm{Ca}^{2+} /$ calmodulin-dependent translocation of sphingosine kinase: Role in plasma membrane relocation but not activation. Cell Calcium 33: 119-128. 


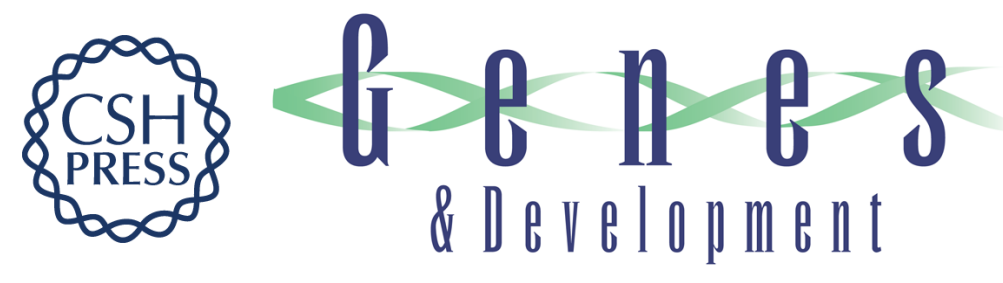

\section{Recruitment of sphingosine kinase to presynaptic terminals by a conserved muscarinic signaling pathway promotes neurotransmitter release}

Jason P. Chan, Zhitao Hu and Derek Sieburth

Genes Dev. 2012, 26:

Access the most recent version at doi:10.1101/gad.188003.112

Supplemental http://genesdev.cshlp.org/content/suppl/2012/05/14/26.10.1070.DC1

Material

References This article cites 85 articles, 30 of which can be accessed free at:

http://genesdev.cshlp.org/content/26/10/1070.full.html\#ref-list-1

License

Email Alerting Receive free email alerts when new articles cite this article - sign up in the box at the top

Service right corner of the article or click here.

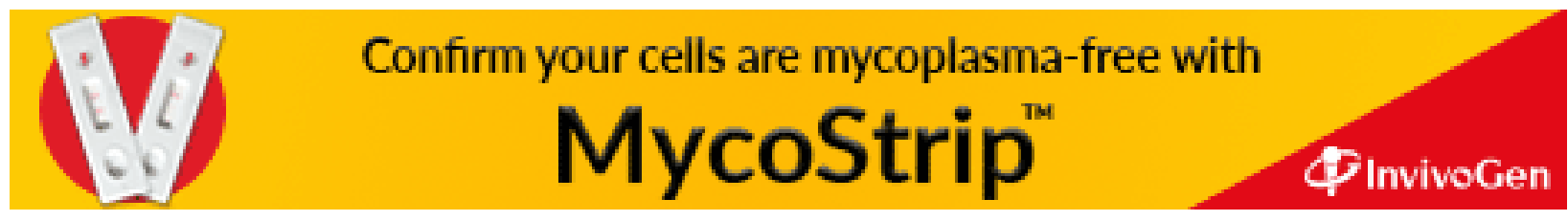

